\title{
Model computations of blue stragglers and W UMa-type stars in globular clusters ${ }^{\star}$
}

\begin{abstract}
K. Stępień and M. Kiraga
Warsaw University Observatory, Al. Ujazdowskie 4, 00-478 Warsaw, Poland

e-mail: [kst, kiraga]@astrouw.edu.pl

Received 19 December 2014 / Accepted 13 March 2015

\section{ABSTRACT}

Context. It was recently demonstrated that contact binaries occur in globular clusters (GCs) only immediately below turn-off point and in the region of blue straggler stars (BSs). In addition, observations indicate that at least a significant fraction of BSs in these clusters was formed by the binary mass-transfer mechanism.

Aims. The aim of our present investigation is to obtain and analyze a set of evolutionary models of cool, close detached binaries with a low metal abundance, which are characteristic of GC.

Methods. We computed the evolution of 975 models of initially detached, cool close binaries with different initial parameters. The models include mass exchange between components as well as mass and angular momentum loss due to the magnetized winds for very low-metallicity binaries with $Z=0.001$. The models are interpreted in the context of existing data on contact binary and blue straggler members of GCs. The models are based on our recently developed code for evolutionary modeling of cool close and contact binaries.

Results. The model parameters agree well with the observed positions of the GC contact binaries in the Hertzsprung-Russell diagram, and we achieve detailed parameters of several individual contact and near-contact binaries. Contact binaries in the lower part of the cluster main sequence are absent because there are no binaries with initial orbital periods shorter than 1.5 d. Contact binaries end their evolution as mergers that appear in the BS region. Binary-formed BSs populate the whole observed BS region in a GC, but a gap is visible between low-mass mergers that are concentrated along the zero-age main sequence and binary BSs occupying the red part of the BS region. Very few binary mergers are expected to rotate rapidly and/or possess chemical peculiarities resulting from the exposure of the layers processed by $\mathrm{CNO}$ nuclear reactions. All other binary mergers are indistinguishable from the collisionally formed mergers. The results show that binary-formed BSs may constitute at least a substantial fraction of all BSs in a GC.
\end{abstract}

Key words. binaries: close - stars: evolution - stars: low-mass - blue stragglers - globular clusters: general - binaries: eclipsing

\section{Introduction}

W UMa-type stars are solar-type eclipsing variables with nearly sinusoidal light curves in which both binary components share a common envelope that is located between the inner and outer critical surfaces (equipotential zero-velocity surfaces crossing the L1 and L2 Lagrangian points), also called inner and outer Roche lobes (Mochnacki 1981). The orbital periods of W UMatype stars are concentrated around 0.25-0.6 d. Because of the physical properties of $\mathrm{W}$ UMa-types stars, we also call them contact binaries (CB).

Numerous W UMa-type stars are observed in globular and old open clusters (Rucinski 1998, 2000), but they are completely absent among $\mathrm{T}$ Tauri stars and in young clusters, even though they are easy to detect (Mathieu 1994; Melo et al. 2001; Hebb et al. 2010). None is known in the intermediate-age cluster Hyades, in spite of the high degree of completeness achieved in the binary search by Griffin (1985), and only one certain W UMa-type member is identified in Praesepe (Rucinski 1998). These data indicate that it takes several Gyr - up to the age of a globular cluster (GC) - before a $\mathrm{CB}$ is formed from its progenitor. The age of several Gyr also results from the kinematic analysis of field W UMa-type stars (Guinan \& Bradstreet 1988; Bilir et al. 2005).

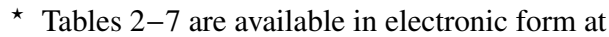
http: //wwW . aanda.org
It is generally accepted that progenitors of CBs are cool detached binaries. In the course of evolution they lose angular momentum (AM) by a magnetized wind blowing from one or both components. With synchronous rotation, stellar AM loss (AML) results in a tightening of the orbit until the primary fills its inner Roche lobe and Roche-lobe overflow (RLOF) occurs. For a binary whose initial orbital period is close to $2 \mathrm{~d}$ and whose initial primary is similar to the Sun, the time to reach RLOF is approximately equal to the evolutionary life time of the primary on the main sequence (MS) (Stępień 2011a). For shorter initial periods and/or lower primary mass, the MS life time is longer. Nonetheless, as long as the initial orbital period is not very different from $2 \mathrm{~d}$, the solar type initial primary is already advanced in evolution when RLOF occurs.

W UMa-type stars observed in GCs always lie close to the turn-off (TO) in the Hertzsprung-Russell diagram (HRD), with a significant fraction falling above $\mathrm{TO}$, in the region of blue straggler stars (BSs), which are stars lying on the high-mass extension of the cluster MS (Rucinski 2000). Early observations of GCs were not sufficiently sensitive to detect CBs in the lower part of the cluster MS, but the recent deep surveys of a few GCs revealed an absence of CBs fainter than about one magnitude below TO (Kaluzny \& Thompson 2009; Kaluzny et al. 2010, 2013a). As Kaluzny et al. (2013a) stressed, not a single system was detected in over 12000 stars in the M4 cluster with 
magnitudes $17.8<V<21$, that is, between one and four magnitudes below TO, whose light curves were analyzed.

The aim of our present investigation is to obtain and analyze a set of evolutionary models of cool, close, detached binaries with a low metal abundance, which is characteristic of GC. Depending on the specific initial conditions, a fraction of the investigated binaries changes into $\mathrm{CBs}$ at the later age. Evolutionary computations are based on a model developed by one of us (Stępień 1995, 2006a, 2009; Gazeas \& Stępień 2008). Altogether, about $10^{3}$ models have been computed with an initial primary mass between $0.7-0.9 M_{\odot}$, an initial secondary mass between $0.4-0.8 M_{\odot}$, an initial orbital period between $1.5-2.9 \mathrm{~d}$, and a metal abundance $Z=0.001$. The results are compared with the available observations of GCs, in particular with the data on the cluster $\mathrm{CBs}$ and BSs.

The next section presents the essentials of the evolutionary model, including the recent improvements of the computer program, which follow the evolution of both components more accurately than in the original code (in particular, the slow masstransfer rate is now calculated every time step), and additional effects are included. The third section describes the models together with the statistical properties of the cool close binary population as it evolves from the beginning to an age of 13.5 Gyr. Bulk properties of binaries that form mergers or are in a contact state at an age of 11, 12, and 13 Gyr are discussed. The observed properties of CBs and BSs, including individual objects from different GCs, are compared with the model predictions in the fourth section. The last section summarizes and discusses the results of the paper.

\section{Description of an evolutionary model of cool close binaries}

\subsection{Equations}

The cool close binary model (CCBM) describes the evolution of a cool close binary from the ZAMS untill a stage immediately preceding the merger of the components or the formation of an Algol-type star with an orbital period of several days. We do not follow the detailed evolution of the Algol-type binary here because it does not fulfill some of the CCBM assumptions. In addition, the Algol and post-Algol evolution has been investigated thoroughly in the past and is well known (e.g., Paczyński 1971; Sarna 1993; Nelson \& Eggleton 2001).

As an initial close binary we took a detached system with an orbital period short enough to ensure full synchronization of the orbital and rotational periods. Because we are mostly interested in progenitors of CBs, we discuss only binaries with initial periods shorter than $3 \mathrm{~d}$. Longer period models always evolve into Algols. Cool means here that both components possess subphotospheric convection zones that generate magnetic activity, in particular, a magnetized stellar wind. An approximate upper mass limit for these stars is about $1.3 M_{\odot}$, although somewhat more massive stars can also be considered because they later develop a convection zone during their MS life. We here focus on stars with initial masses equal to or lower than $0.9 M_{\odot}$. They have a sufficiently deep convection zone already on the ZAMS. We apply the Roche model to describe the orbital parameters.

The basic equations of CCBM are the third Kepler law, the expression for AM, and the approximate expressions for inner Roche-lobe sizes $r_{1}$ and $r_{2}$ Eggleton (1983),

$P=0.1159 a^{3 / 2} M^{-1 / 2}$,

$H_{\text {tot }}=H_{\text {spin }}+H_{\text {orb }}$, where

$H_{\text {spin }}=7.13 \times 10^{50}\left(k_{1}^{2} M_{1} R_{1}^{2}+k_{2}^{2} M_{2} R_{2}^{2}\right) P^{-1}$,

and

$$
\begin{aligned}
& H_{\text {orb }}=1.24 \times 10^{52} M^{5 / 3} P^{1 / 3} q(1+q)^{-2}, \\
& \frac{r_{1}}{a}=\frac{0.49 q^{2 / 3}}{0.6 q^{2 / 3}+\ln \left(1+q^{1 / 3}\right)}, \\
& \frac{r_{2}}{a}=\frac{0.49 q^{-2 / 3}}{0.6 q^{-2 / 3}+\ln \left(1+q^{-1 / 3}\right)}
\end{aligned}
$$

Here $P$ is the period (rotational and orbital), $M=M_{1}+M_{2}$ is the total mass with component masses $M_{1}$ and $M_{2}, R_{1}$ and $R_{2}$ are component radii, $a$ is the semi-axis, $H_{\text {tot }}, H_{\text {spin }}$ and $H_{\text {orb }}$ are total, rotational and orbital AM, $k_{1}^{2}$ and $k_{2}^{2}$ are the (nondimensional) gyration radii of the two components, $r_{1}$ and $r_{2}$ are the sizes of the inner Roche lobes, and $q=M_{1} / M_{2}$ is the mass ratio. Masses, radii, and semi-axis are given in solar units, the period is given in days, and the AM in cgs units.

We assumed that stellar winds from the two components are the dominating mechanism of the orbit evolution. They carry away mass and AM according to the formulas

$$
\begin{aligned}
& \dot{M}_{1,2}=-10^{-11} R_{1,2}^{2}, \\
& \frac{\mathrm{d} H_{\mathrm{tot}}}{\mathrm{d} t}=-4.9 \times 10^{41}\left(R_{1}^{2} M_{1}+R_{2}^{2} M_{2}\right) / P .
\end{aligned}
$$

Here $\dot{M}$ is in solar mass per year and $\mathrm{d}_{\text {tot }} / \mathrm{d} t$ is in $\mathrm{g} \mathrm{cm}^{2} \mathrm{~s}^{-1}$ per year. The formulas were discussed at length by Stępień (2006a,b) and Gazeas \& Stępień (2008). They are calibrated by the observational data of the rotation of single, magnetically active stars and empirically determined mass-loss rates of single, solar type stars. Both formulas apply in a limiting case of a rapidly rotating star in the saturated regime. Note that they do not contain any free adjustable parameters. The constant in Eq. (7) is uncertain within a factor of 2 and that in Eq. (8) is uncertain to $\pm 30 \%$ (Stępień 2006b; Wood et al. 2002). Any interaction between winds from the two components is neglected.

\subsection{Initial assumptions}

The model equations were applied to binaries with parameters characteristic of GC members. The CCBM evolution was followed until the age of the oldest GCs and snapshots of the model population properties are discussed in detail for three specified ages of 11, 12 and 13 Gyr. We focused on stars with initial masses close to the TO mass, because more massive cluster members have already completed their evolution and have formed compact objects, whereas the less massive ones are still on the MS, burning hydrogen in their cores. Specifically, we took initial primary masses from the interval $0.7-0.9 M_{\odot}$, with the detailed values given in Table 1 . If the primary in a model has a mass that is closely spaced between $0.86-0.87 M_{\odot}$, it means that the primaries reach RLOF between 11 and 13 Gyr. The initial masses of the secondary component were adjusted to avoid extreme mass ratios $q_{\text {init }}=1$ and $q_{\text {init }} \gg 1$. The evolution of binaries with exactly equal mass components is uninteresting to us, whereas the rapid mass transfer following RLOF is very likely nonconservative for high values of the mass ratio. Without a reliable theory describing this process, the standard procedure is to use free parameters for mass and AM losses (Sarna \& De Greve 1996). To avoid introducing them, we assumed conservative mass transfer. Our models have $1.08 \leq q_{\text {init }} \leq 2.25$. The masses 
Table 1. Initial component masses of the computed binary models.

\begin{tabular}{ll}
\hline \hline$M_{1}\left(M_{\odot}\right)$ & $M_{2}\left(M_{\odot}\right)$ \\
\hline 0.7 & $0.4,0.5,0.6$ \\
0.75 & $0.4,0.5,0.6$ \\
0.8 & $0.4,0.5,0.6,0.7$ \\
0.81 & $0.4,0.5,0.6,0.7$ \\
0.82 & $0.4,0.5,0.6,0.7$ \\
0.83 & $0.4,0.5,0.6,0.7$ \\
0.84 & $0.4,0.5,0.6,0.7$ \\
0.85 & $0.4,0.5,0.6,0.7$ \\
0.86 & $0.4,0.5,0.6,0.7$ \\
0.865 & $0.4,0.5,0.6,0.7,0.8$ \\
0.8675 & $0.4,0.5,0.6,0.7,0.8$ \\
0.87 & $0.4,0.5,0.6,0.7,0.8$ \\
0.88 & $0.4,0.5,0.6,0.7,0.8$ \\
0.89 & $0.4,0.5,0.6,0.7,0.8$ \\
0.9 & $0.4,0.5,0.6,0.7,0.8$ \\
\hline
\end{tabular}

Notes. For each combination of $M_{1}$ and $M_{2}$ a set of 15 evolutionary models has been calculated with initial orbital periods $1.5,1.6, \ldots, 2.8$, $2.9 \mathrm{~d}$.

of the secondary component are also given in Table 1. For each pair of the component masses, 15 models were computed with initial period lengths from $1.5 \mathrm{~d}$ to $2.9 \mathrm{~d}$, every $0.1 \mathrm{~d}$. Altogether, 975 evolutionary models were obtained. In the following, we identify individual models by giving the initial values of three basic parameters: $M_{1}+M_{2}(P)$, for instance, $0.8+0.6(1.8)$.

We adopted the range of initial period values according to the following considerations. It is now generally accepted that binary and multiple stars are formed during the early fragmentation of a protostellar cloud (Boss 1993; Bonnell 1994; Kratter et al. 2010; Machida et al. 2008). After the end of the rapid accretion phase, cool protostars enter the $\mathrm{T}$ Tauri phase when the mass has almost reached its final value and changes only little. This transition takes place at the age of $10^{5}-10^{6}$ years (Machida et al. 2010; Vorobyov 2010; Ohtani \& Tsuribe 2013). The orbit of a binary with two freshly formed T Tauri-type components must be wide enough to accommodate both stars. The youngest $\mathrm{T}$ Tauri stars with masses between 0.5 and $1.5 M_{\odot}$ have radii from 2 up to over 3 solar radii (Baraffe et al. 1998; Tognelli et al. 2011), which means that the initial orbital period must be longer than about $1.5 \mathrm{~d}$ (the accurate value depends only weakly on the mass ratio). We adopted this value as a lower limit for the initial orbital period of an isolated binary. Shorter period binaries can be formed by dynamical interactions (collisions) with other objects in a GC (Heggie 1975; Hills 1975; Hut 1983; Leigh et al. 2013; Hypki \& Giersz 2013), but we excluded them from our analysis.

There exists another efficient mechanism that effectively shortens the orbital periods of some binaries. It requires a third body on a very wide and strongly inclined orbit around the inner binary. The third companion induces so-called Kozai cycles that periodically change the eccentricity of the inner orbit. Tidal interactions between the components during periastron passages dissipate the system energy and tighten the orbit. Numerical models of this mechanism, called Kozai cycles with tidal friction (KCTF), show that a significant fraction of binaries with initial periods of 2-3 weeks shorten their periods to 2-3 d within several million years (Eggleton \& Kiseleva-Eggleton 2006; Fabrycky \& Tremaine 2007; Perets \& Fabrycky 2009; Naoz \& Fabrycky 2014). At these periods the orbits circularize and do not change any more (Fabrycky \& Tremaine 2007).
Population synthesis indicates that among binaries with periods shorter than $3 \mathrm{~d}$, those formed by the KCTF mechanism outnumber isolated systems (Fabrycky \& Tremaine 2007). Observations of field binaries confirm this expectation. Tokovinin et al. (2006) found out that $96 \%$ of binaries with periods shorter than $3 \mathrm{~d}$ possess a third companion, in contrast to $34 \%$ of binaries with periods longer than $12 \mathrm{~d}$. A similar conclusion was reached by Rucinski et al. (2007), who showed that all or almost all field W UMa-type binaries possess a tertiary component.

Consequently, we assumed that the initial period distribution of cool close binaries has a sharp maximum at $2-3 \mathrm{~d}$, resulting mostly from the KCTF mechanism, and a rapid decline toward $1.5 \mathrm{~d}$. The extension of the distribution beyond $3 \mathrm{~d}$ is unimportant for our purposes because, as we show below, these binaries do not form CBs.

\subsection{Description of evolutionary computations}

The evolution of cool close binaries can be divided into three phases (Stępień 2006a; Paczyński et al. 2007; Gazeas \& Stępień 2008), where phase I begins at the initial state to RLOF by the initial primary (denoted with the subscript " 1 " throughout, also when it becomes a less massive component). The other component is marked " 2 ". The binary is detached in phase I, and its evolution is dominated by two mechanisms: individual evolution of each component across the MS, and the magnetized winds carrying away mass and AM. Any interaction between winds from the two components is neglected, and no other mechanism influencing the orbit is considered. Equations (1)-(8) are integrated at every time step. The typical length of the time step was set to $6.8 \times 10^{7}$ years so that the age of a GC is reached in about 200 steps. At each time step, the radii of the components were calculated using the grid of single-star models with a metal content $Z=0.001$ published by Girardi et al. (2000). The value of the radius was interpolated in time and mass. The total mass lost during phase I amounts to several percent of the initial stellar mass, depending on stellar radius and the duration of phase I. This has a minor effect on the radius evolution of the components. More important is the evolutionary advancement due to hydrogen burning, which slowly inflates a star. At the same time, the Roche lobes descend onto the stellar surfaces due to AML (Eq. (8)). If RLOF did not occur within $13.5 \mathrm{Gyr}$, calculations were stopped and the binary remained detached. To allow for the supersaturation of magnetic activity in ultra-fast rotators (Randich et al. 1996; Prosser et al. 1996; Stępień et al. 2001), the period in Eq. (8) was replaced by $0.4 \mathrm{~d}$ for binaries with orbital periods shorter than that limiting value. Phase $I$ is the longest evolutionary phase with a duration of the same order as the MS lifetime of the initial primary.

Following RLOF, phase II begins when a rapid mass transfer occurs from the initial primary (henceforth loser) to the initial secondary (gainer). It is much shorter than other phases and lasts no more than one or a few percent of phase I. The details of this process are discussed by Stępień \& Kiraga (2013a). The gainer swells after receiving a small amount of matter, until it fills its inner Roche lobe and the system temporarily assumes a contact configuration (Webbink 1976). The mass that then flows from the loser encircles the swollen gainer close to its equator and returns to the parent star (Stępien 2009). With no mass accreted, the swollen star slowly shrinks, trying to revert to thermal equilibrium. When its surface moves below the Roche lobe, the star can again receive a small amount of mass, which causes it to expand back to the size of the lobe. The process of shrinking, gaining some mass, and expanding again 
is repeated (a sort of equilibrium is established where an instantaneous mass-accretion rate just balances swelling and shrinking of the star close to the Roche lobe), resulting in a masstransfer rate governed by the thermal timescale of the gainer rather than loser. As soon as the thermal timescale of the loser becomes longer than that of gainer (this occurs when the two components have approximately equal masses), the gainer can receive all the transferred mass. Phase II ends when both stars regain equilibrium and the loser just fills its Roche lobe. The mass ratio is inverted so that the initial primary is now less massive than the initial secondary. Regardless of the details of the mass transfer process, we assumed that mass is transferred on a thermal timescale of $\sim 10^{8}$ years. A constant mass transfer rate $\dot{M}_{\mathrm{tr}}=5 \times 10^{-9} M_{\odot} \mathrm{yr}^{-1}$ was adopted based on observation that the total mass transferred during phase II in any of the modeled binary did not exceed $0.5 M_{\odot}$.

In most cases, a short-period semi-detached configuration of the Algol-type was obtained at the end of phase II with the gainer inside its Roche lobe. We call this a near contact binary (NCB). But a few binaries were so compact that a CB was already formed before phase III began.

Similarly as in phase I, the evolutionary expansion of both stars, accompanied by mass and AM loss by the winds, drives the binary evolution in phase III. In particular, the expansion of the loser beyond its Roche lobe results in a slow mass transfer (on the evolutionary timescale) to the gainer. This widens the orbit, which in turn suppresses the transfer. In absence of winds, the binary period systematically increases until mass transfer stops completely. In the presence of a low AML rate, the period still increases, although at a lower pace. If the AML rate is high and/or the mass transfer rate low, however, the orbit shrinks until both components merge. Each binary follows one of these routes, depending on the relative efficiency of the two processes.

To compute the binary evolution in phase III, we used the same equations and assumptions as in phase I. Particularly important was a proper treatment of the loser. Because we lack detailed evolutionary models of binary components after a rapid mass exchange, single-star models were used, as described above. Analytic formulas were fitted to mass-radius relations of stars at different evolutionary stages: at the MS, at the TAMS, and for several values of the helium core mass. In addition, formulas for time derivatives of stellar radii were found for different masses and evolutionary stages. Using them, the radius of the secondary component was computed at each time step and compared to the Roche lobe size. The mass transfer rate resulted from this comparison.

Several different formulas for $\dot{M}_{\text {tr }}$ following RLOF in cool close binaries have been used in the past assuming conservative mass transfer. Iben \& Tutukov (1984) assumed proportionality of $\dot{M}_{\text {tr }}$ to $\left(R_{1} / r_{1}\right)^{10}$, Eggleton \& Kiseleva-Eggleton (2002) to $\left(\ln \left(R_{1} / r_{1}\right)\right)^{3}$, whereas Han et al. (2000) used

$\dot{M}_{\mathrm{tr}}=C \frac{\left(R_{1}-r_{1}\right)^{\alpha}}{r_{1}}$ for $R_{1}>r_{1}$,

$\dot{M}_{\text {tr }}=0$ for $R_{1} \leq r_{1}$,

with $C=1000 M_{\odot} \mathrm{yr}^{-1}$ and $\alpha=3$. After several trials, we adopted the formula of Han et al., but with $C=1 M_{\odot} \mathrm{yr}^{-1}$ and $\alpha=5$. The adopted values secured stability for most of the models. Only in a few cases unnecessary oscillations appeared (see below).

This method of calculating the mass-transfer rate was applied to semi-detached binaries until the gainer reached its Roche lobe, thus producing a CB. After that, another approach had to be used because both stars overfill their Roche lobes. Now, the degree of overfill by the gainer was calculated at every time step and compared with the same quantity for the loser. If the former was lower than the latter, the mass transfer rate was increased according to Eq. (9), except that an upper limit of $2 \times 10^{-9} M_{\odot} \mathrm{yr}^{-1}$ was imposed to keep the changes of the binary configuration smooth. If not, the zero transfer rate was applied for the current time step. The model calculations were stopped when the degree of the inner Roche-lobe overfill by both stars reached $20 \%$, attaining thus the outer Roche lobe for typical values of the mass ratio of the modeled binaries $(q \approx 0.6-0.8)$. At this moment, the binary was assumed to lose mass and AM through the Lagrangian $\mathrm{L}_{2}$ point at a rapidly increasing rate with an ultimate merging of the components. Similarly, merging should also occur as a result of the Darwin instability when the spin AM exceeds one third of the orbital AM. This occurs for mass ratios below 0.1 (Rasio 1995). We stopped the calculations at this value, notwithstanding the low degree of overfill.

The contact configuration arises from the NCB formed at the beginning of phase III when the influence of AML on the orbit prevails, or at least balances the mass-transfer rate so that the orbit remains tight during phase III. In the opposite situation, the orbit widens, the period increases, and the binary remains in the Algol configuration with the gainer well inside its Roche lobe. This occurs when the initial primary reaches RLOF after completing its MS evolution, so that hydrogen in the central part of its core is already exhausted. Consequently, it moves to the red giant region on the HRD, and its rapid expansion results in a high enough mass-transfer rate to dominate the orbit evolution. We stopped the calculations for orbital periods exceeding $3 \mathrm{~d}$ when the spin-orbit synchronization may already break down.

The spin AM of the two components is about two orders of magnitude lower than the orbital AM for all considered binaries during phases I-II and for almost all binaries during phase III. Nonetheless, they were calculated at each time step. Constant values of gyration radii were adopted: $k_{1}^{2}=0.10$, which corresponds to a star with core hydrogen depleted, and $k_{2}^{2}=0.06$, corresponding to an unevolved MS star (Rutten \& Pylyser 1988; Claret \& Gimenez 1992). The value of $k_{1}^{2}$ is overestimated for early evolutionary phases, but it better reflects the internal structure of a loser in phase III. In addition, the total binary luminosity and the average effective temperature of CCBMs were calculated at each time step. The sum of the component luminosities was adopted as the binary luminosity, and the binary effective temperature was obtained from the surfaceweighted mean brightness of both stars,

$$
\begin{aligned}
& L_{\mathrm{b}}=L_{1}+L_{2}, \\
& T_{\mathrm{eff}, \mathrm{b}}^{4}=L_{\mathrm{b}} / 4 \pi \sigma\left(R_{1}^{2}+R_{2}^{2}\right),
\end{aligned}
$$

where $L_{\mathrm{b}}$ is the binary luminosity and $T_{\mathrm{eff}, \mathrm{b}}$ is the binary effective temperature.

Figure 1 shows the region of the HRD that is occupied by the initial CCBMs. To avoid crowding, only 19 models were plotted with a primary mass between $0.7-0.9 M_{\odot}$, every $0.1 M_{\odot}$, and all secondary masses accompanying them. We also plot the isochrone $t=10^{8}$ years, $Z=0.001$ from the latest Padova models $^{1}$, which shows the approximate position of the ZAMS for a GC. The modeled binaries all lie above the cluster MS, as expected.

http://stev.oapd.inaf.it/cmd 
K. Stępień and M. Kiraga: Blue stragglers and W UMa-type stars in globular clusters

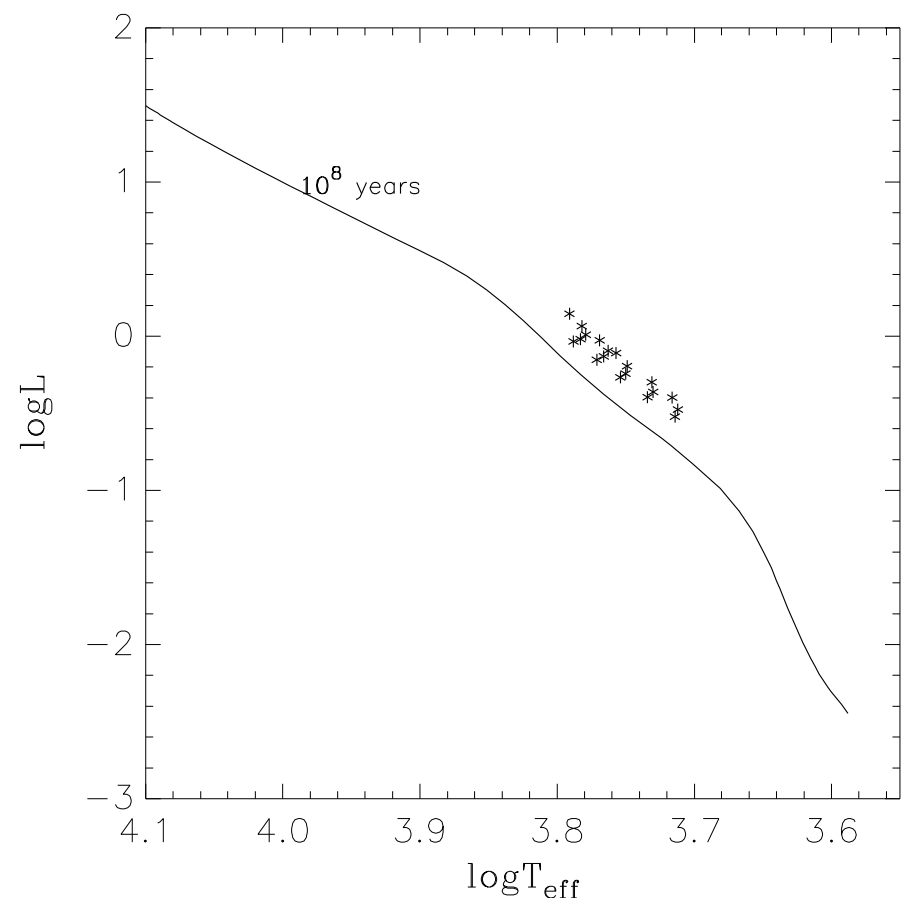

Fig. 1. HR diagram with representative initial binary models plotted together with a low-metal isochrone assumed to track the ZAMS of a GC.

\section{Results}

\subsection{Period evolution}

The binary evolution sensitively depends on whether the primary mass is higher or lower than the TO mass. Primaries with lower masses remain detached untill the specified age of a GC, unless their initial orbital period is significantly shorter than $1.5 \mathrm{~d}$. Figure 2 presents an example of the period evolution of binaries with a primary mass lower than the TO mass. Out of all 60 models with an initial primary mass of $0.8 M_{\odot}, 19(32 \%)$ reach phase III within an age of $13.5 \mathrm{Gyr}$, whereas the others remain detached. Corresponding figures for other low masses look similar, except that the number of models reaching phase III increases from only $4(9 \%)$ for $0.7 M_{\odot}$ to $35(58 \%)$ for $0.86 M_{\odot}$ (note that because of the mass lost through stellar winds, the final masses of the stars are about 6-7\% lower than initial). All binaries with initial primaries more massive than $0.86 M_{\odot}$ reach RLOF within the age of $13.5 \mathrm{Gyr}$ and show pronounced evolutionary effects (see an example in Fig. 3). Here we also see that binaries with periods longer than $\sim 2 \mathrm{~d}$ reach RLOF when their primaries have already left the MS, which results in a high mass-transfer rate in phase III and a rapid period increase. Some models with $q$ approaching 0.1 show spurious period oscillations before merging, see for example the model in the lower right of Fig. 3. This is caused by a too long time step. We used a constant value of $5 \times 10^{6}$ years for all models in phase III, which means that the typical duration of this phase is 200-400 steps. A high instantaneous mass-transfer rate, multiplied by the long time step, results in a rapid decrease of an already low loser mass, producing a sudden period jump, followed by its decrease at the next time step as the mass transfer rate drops to zero. We obtained exemplary calculations for the time step shortened four times, that is, for $1.25 \times 10^{6}$ years. The period oscillations are then completely absent (Fig. 4). Switching to such a short time step would, however, substantially lengthen the computations, therefore we decided to ignore these oscillations in the

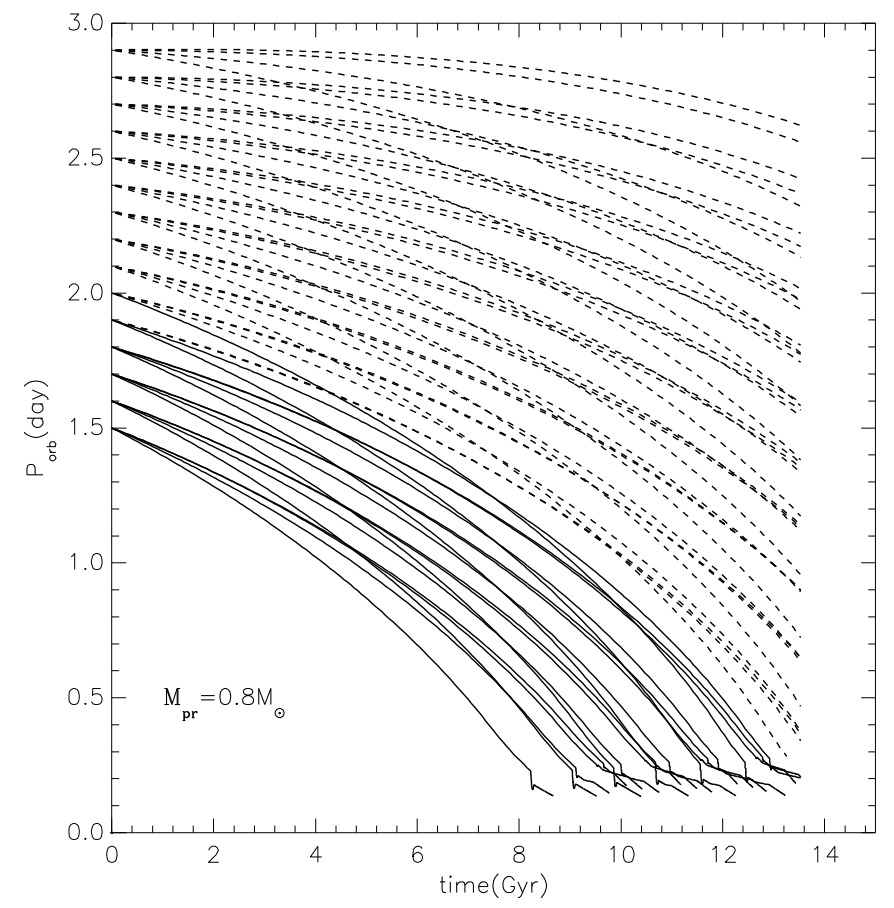

Fig. 2. Variation in time of the orbital period of all binary models with an initial primary mass equal to $0.8 M_{\odot}$. Period variations of binaries that have not yet reached RLOF within the age of the Universe are shown with dotted lines and solid lines describe the period variations of binaries that reach RLOF within this age.

following analysis. We did so because a correct value of a binary period matters only when summation over consecutive bins is performed to obtain the period distribution at a specified age (see below). Instead of trying to determine a correct period value, for instance, by determining the local minimum in the vicinity of this age, we included its instantaneous value in the summation. In effect, a small number of periods fall incorrectly into an adjacent bin. This changes the general appearance of the distribution only little. The components merge as a result of the Darwin instability always near the local period minimum, which is close to the correct value (Fig. 4).

\subsection{Period distribution}

The orbital period distribution of cool close binaries in GC evolves in time under the influence of several mechanisms. Some, such as encounters with other stars, harden the binaries by shortening their periods (Heggie 1975; Hills 1975; Hut 1983; Hypki \& Giersz 2013; Leigh et al. 2013), or like the KCTF mechanism that operates on a timescale of several Gyr because of the very long initial period of the inner binary (Fabrycky \& Tremaine 2007), inject binaries into the considered period interval. Other mechanisms such as expelling binaries from GC, component merging, or formation of Algols with longer periods, remove binaries from that interval. A full discussion of all these mechanisms is beyond the scope of the present paper. We consider here only the evolution of the period distribution of CCBMs.

We made the following assumptions about the population of cool close binaries in a GC. 


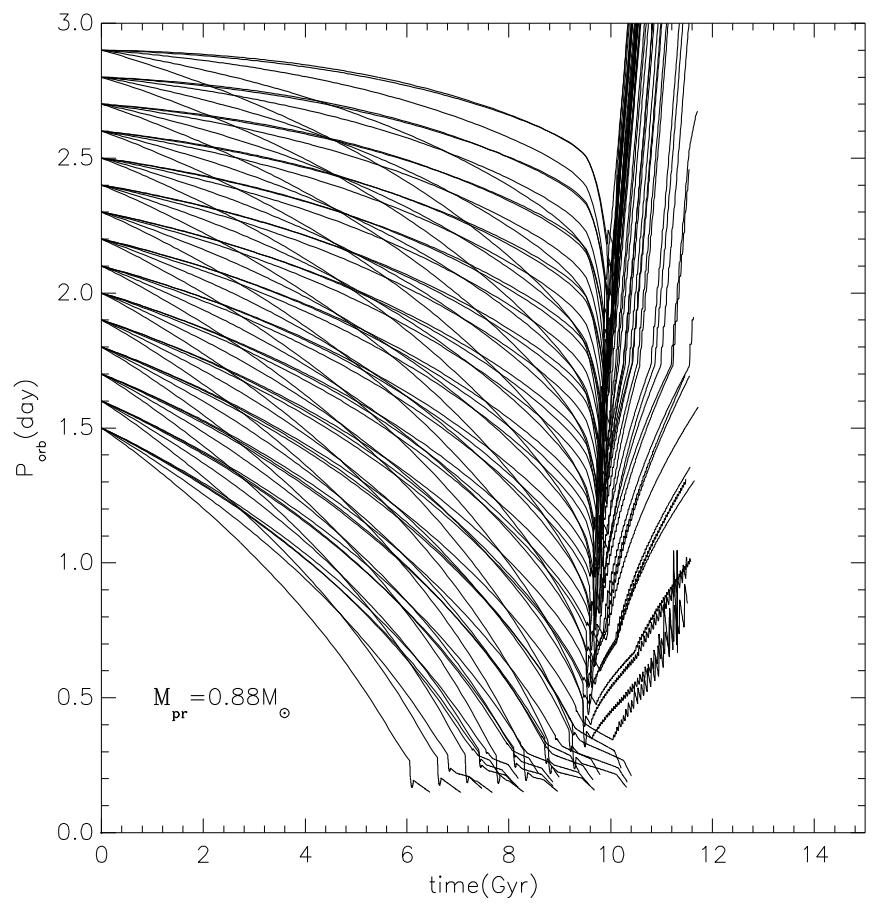

Fig. 3. Variation in time of the orbital period of all binary models with an initial primary mass equal to $0.88 M_{\odot}$. All these binaries reached RLOF within the age of $11 \mathrm{Gyr}$.

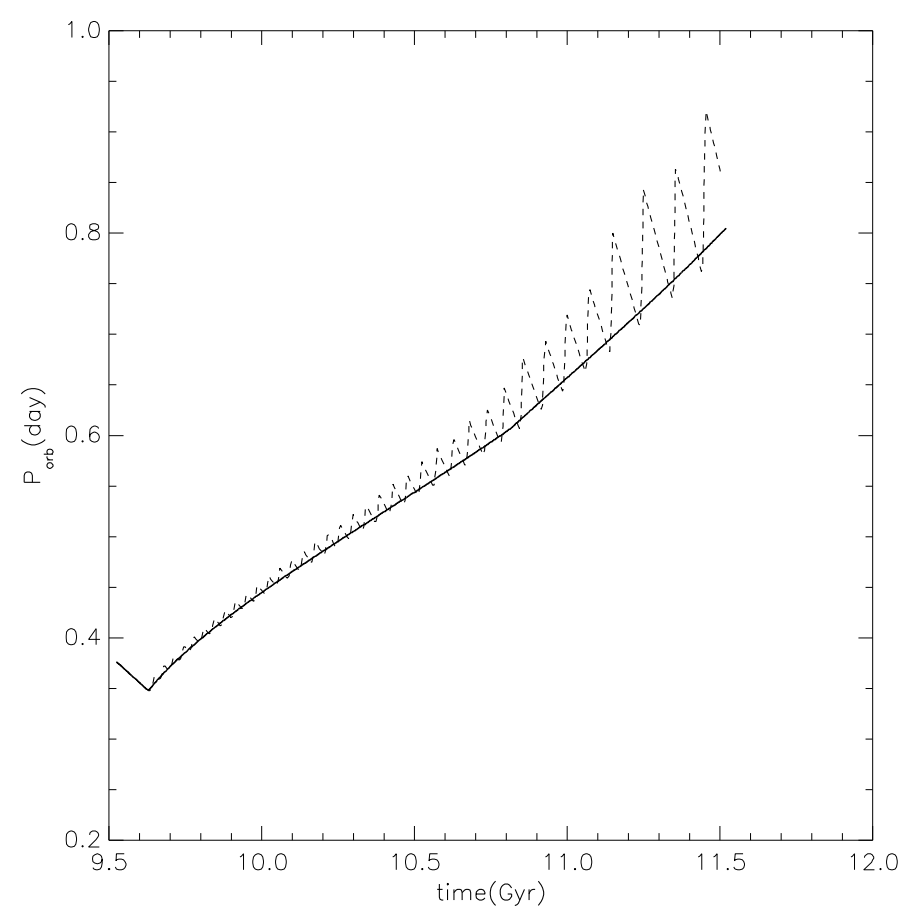

Fig. 4. Period variations of a model $0.88+0.4(2.2)$ in phase III, calculated with a time step of $5 \times 10^{6}$ years (dotted line) and $1.25 \times 10^{6}$ years, i.e. four times shorter (solid line).

1. The initial period distribution is constant for the period interval $2.0-2.9 \mathrm{~d}$ and decreases linearly to zero at $1.4 \mathrm{~d}$ :

$$
\begin{aligned}
& n\left(P, M_{1}, q\right) \mathrm{d} P=0 \text { for } P<1.4, \\
& n\left(P, M_{1}, q\right) \mathrm{d} P=\frac{5}{3}(P-1.4) \text { for } 1.4 \leq P<2, \\
& n\left(P, M_{1}, q\right) \mathrm{d} P=1 \text { for } P \geq 2,
\end{aligned}
$$

where $n\left(P, M_{1}, q\right)$ is the number density of binaries with different periods and mass ratios. The value of 1.4 results from a linear approximation of the step-wise distribution considered here, where a first non-zero value of $n$ is for $P=1.5 \mathrm{~d}$. The lack of shorter initial periods is due to the inflated component radii during the T Tauri phase (see Sect. 2.2).

2. The initial period and component masses are not correlated, that is, $n\left(P, M_{1}, q\right) \mathrm{d} P \mathrm{~d} M \mathrm{~d} q=n_{1}(P) n_{2}\left(M_{1}\right) n_{3}(q) \mathrm{d} P \mathrm{~d} M \mathrm{~d} q$, where $n_{1}(P), n_{2}\left(M_{1}\right)$ and $n_{3}(q)$ are the number densities depending on period, mass, and mass ratio, respectively. This follows from the semi-empirically determined properties of the birth binary population (Kroupa 1995).

3. The primary mass distribution is given by the initial mass function of Salpeter (1955): $n_{2}\left(M_{1}\right) \propto M_{1}^{-2.35}$. Alternative forms of this function have also been proposed by Miller \& Scalo (1979) and Scalo (1986). More recently, Kroupa (2002) confirmed the Salpeter mass function for stars with masses higher than $0.5 M_{\odot}$, except that he suggested a somewhat flatter mass dependence with an exponent of 2.3. Nevertheless, the original Salpeter relation is still in use. The difference between both exponents has a negligible influence on the mass function over the narrow mass interval considered here.

4. The mass ratio distribution is flat, $n_{3}(q)=$ const. (Raghavan et al. 2010; Milone et al. 2012), although short-period field binaries may show some preference to $q \approx 1$ (Duchêne $\&$ Kraus 2013). In lack of detailed information on the mass ratio distribution of GC binaries, we adopted the flat distribution.

Before calculating the period distribution, a weight for each CCBM must be determined to be used in the binary summation. The initial CCBMs are distributed uniformly in $P$, which means that each model represents binaries from an equal period interval. When summation is performed over consecutive period bins, the weight $w_{P}$, attached to each model, is therefore constant for $P \geq 2 \mathrm{~d}$ and decreases linearly to zero, as given by Eq. (12). The situation is more complicated when we consider weights $w_{M}$ and $w_{q}$ connected with the initial primary mass and initial mass ratio, because the models are distributed nonuniformly in $M_{1}$ and $q$. To obtain their values, it was necessary first to calculate the widths of the respective intervals in $M_{1}$ and $q$, centered on each model, and then to multiply them by factors resulting from assumptions 3 and 4 above. Finally, a product of all three weights was attached to each of the 975 CCBMs and kept constant over all evolutionary calculations.

Figure 5 presents the time evolution of the period distribution with the $0.1 \mathrm{~d}$ bin size. The evolving distribution is shown every $1 \mathrm{Gyr}$, starting from the initial distribution at the bottom of the figure untill the age of 13 Gyr. The last distribution, at the top of the figure, corresponds to the age of 13.5 Gyr. Broken lines show zero lines of the consecutive distributions.

The main evolutionary effects of the period distribution consist of the shift of the short-period limit toward the shorter periods, together with a stretching or flattening of the distribution, except for its long-period end, where a deficit of binaries is visible. The deficit is due to the period increase of several binaries beyond the three-day limit, which is not compensated for by the possible period shortening of binaries with initial periods $\geq 3 \mathrm{~d}$. They were not included in the present models, but we can estimate their approximate influence on the plotted distribution. All binaries with initial periods 3-3.5 d shorten them below $3 \mathrm{~d}$ during phase I, but those with primaries more massive than the TO mass quickly increase their periods above $3 \mathrm{~d}$ 


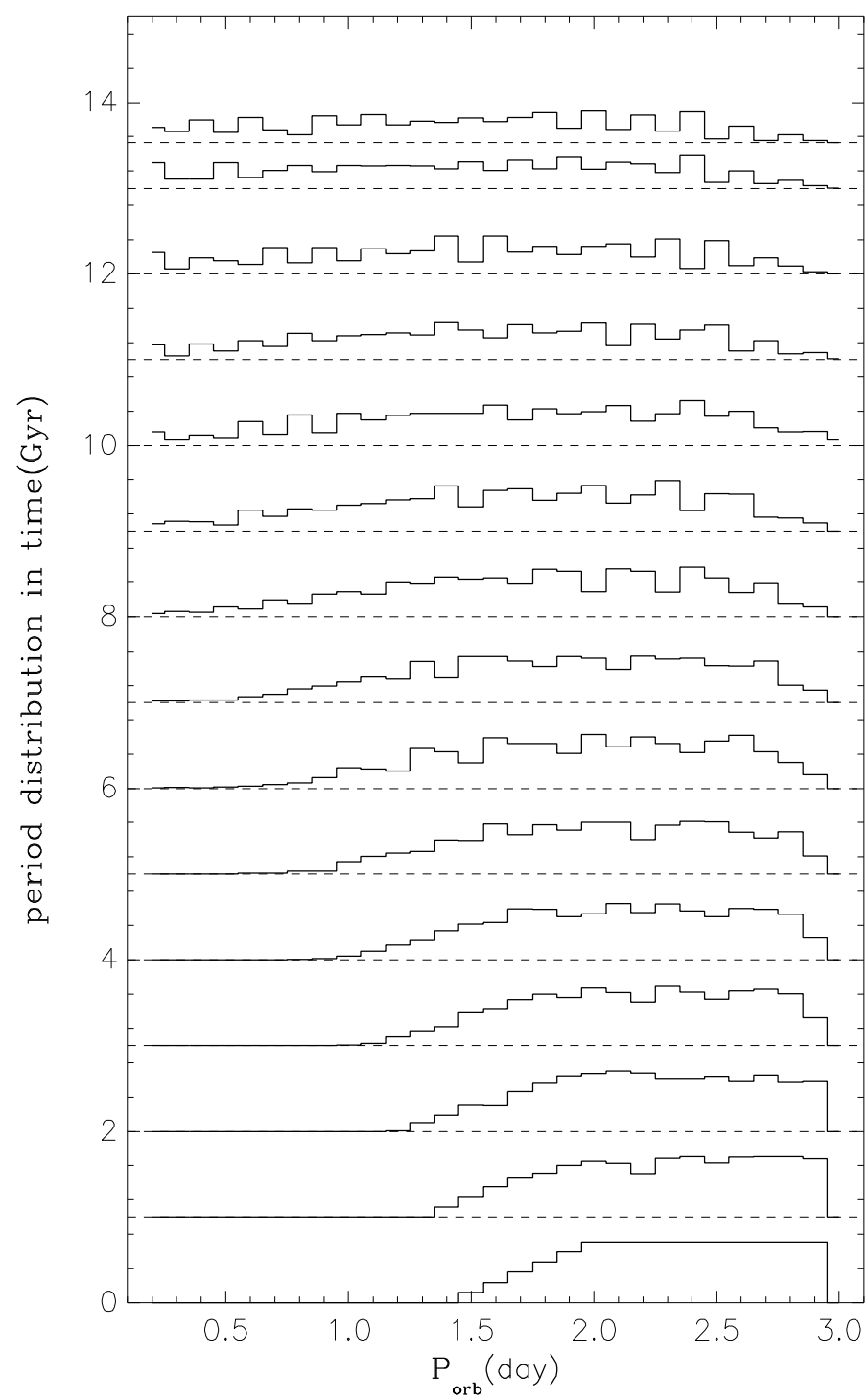

Fig. 5. Evolution of the orbital period distribution of cool close binaries in time. Distributions are shown every $1 \mathrm{Gyr}$ from 0 till $13 \mathrm{Gyr}$, and they are supplemented with the distribution at $13.5 \mathrm{Gyr}$. The ordinate gives the age of the binary population in Gyr.

past RLOF (similarly as shown in Fig. 3). Lower mass binaries with the same initial periods remain detached over the Hubble time and shorten their periods continuously, but lose only about $15-20 \%$ of the initial AM because the AML rate is inversely proportional to the period, see Eq. (8). Their final periods fall between 2.4-3 d, so they would partly fill in the deficit if they are included in the distribution. Nontheless, a comparison between the observed and predicted distribution should be limited to periods shorter than $2.4 \mathrm{~d}$. Binaries with initial periods above 3.5 never enter the period interval plotted in Fig. 5. This means that the distribution does not go to zero at the long-period boundary, but its exact shape there depends on the initial period distribution beyond $3 \mathrm{~d}$, which is not considered.

The general properties of the period distribution are insensitive to the bin size, for example, increasing the bin size twice results in a less ragged shape, but the broadening toward short periods and flattening of the distribution are still clearly visible.

The short-period limit crosses the $1 \mathrm{~d}$ mark at the age of $4 \mathrm{Gyr}$ and $0.5 \mathrm{~d}$ mark at about $7 \mathrm{Gyr}$. This is a very interesting result. It shows that the value of the short-period limit of detached MS binaries in a stellar cluster can be used as an independent measure of the cluster age, similarly as the rotation period of cool MS stars is a measure of stellar age (Barnes 2009; Meibom et al. 2011). At present, very few clusters are known with a sufficiently complete sample of binary stars to safely determine a short-period limit. However, the increasing accuracy of photometric observations in the future will enable detecting light variations caused by the ellipticity of noneclipsing binary components. As a result, a high percentage of all close binaries will be detected in several clusters and a short-period limit of the period distribution can be accurately determined. Since close binaries can also be formed by collisions with other cluster members (ignored here), their possible effects on this limit will have to be quantified before the method is applied to a particular cluster.

The flattening of the distribution with time is accompanied by a decrease of the total number of binaries within the considered period interval that is due to two mechanisms: merging of the two components, and a period increase beyond $3 \mathrm{~d}$ of Algoltype binaries. As a result, the total weighted number of CCBMs (equivalent to the integral of the distribution over period) in the period interval 0.15-3.0 d decreases by about one third (more accurately, by $32.9 \%$ ) at $13.5 \mathrm{Gyr}$, compared to the initial data. Unweighted number of models included in the distribution decreased at that time from an initial 975 binaries to 398 (41\%).

We conclude that the predicted shape of the period distribution of cool close binaries at the age of a GC is flat over the interval $0.1-2.5 \mathrm{~d}$ and bears no similarity to the initial distribution.

\subsection{Mergers and contact binaries in a globular cluster}

As we showed above, the binary evolution past RLOF depends on the relative importance of slow mass transfer that lengthens the orbital period and of the AML that shortens it. Binaries with rapidly increasing period remain in an Algol configuration, whereas the rest experience a contact phase with the ultimate merging of the components if the timescale for a merger is shorter than the cluster age.

The model calculations show that coalescence typically occurs in less than $1 \mathrm{Gyr}$ after RLOF, and the typical lifetime of a merger in the region of BS is also of the order of 1 Gyr. We therefore focused on binaries with a RLOF occurring at, or later than, $10 \mathrm{Gyr}$, which then evolve toward a CB or NCB configuration. Binaries evolving to the Algol configuration are not discussed.

We selected model binaries in which RLOF occurred between 10 and $11 \mathrm{Gyr}$ and which have orbital periods shorter than $1 \mathrm{~d}$ at the age of $11 \mathrm{Gyr}$. In many of them (particularly those with the shortest periods), both components already fill their Roche lobes, but in others only the loser does, whereas the gainer is within its lobe. The computed stellar radii result strictly from the evolutionary models without allowing for any effects that might influence them (e.g., magnetic fields or deviations from thermal equilibrium). The observed radii of cool, rapidly rotating stars are often larger than the model radii, so several real stars with the same global parameters as CCBMs may in fact be already CBs even if they look like NCBs in our simulations. We marked binaries with $P<0.5 \mathrm{~d}$ as $\mathrm{CBs}$ and those with $1>P \geq 0.5 \mathrm{~d}$ as NCBs and indicate them with asterisks and plus signs, respectively. Numerical data on these models are given in Table 2 with the consecutive columns giving the name of the model, component masses, mass ratio, period, luminosity, effective temperature, and remarks: $\mathrm{CB}$ - contact binary, NCB - near-contact binary, MS - binary lies below TO, $\mathrm{BS}$ - binary lies in the BS region. The same data are plotted in 
Fig. 6 together with the ZAMS and a $11 \mathrm{Gyr}$ isochrone. In addition, the red boundary of the BS region we adopted is shown as the long-dashed line. This is the ZAMS line shifted upward by $\Delta \log L=0.8$ (=2 mag). It lies close to the TAMS line for the most massive BSs and somewhat higher for TO stars (Schaller et al. 1992). A similar red boundary was adopted by Leigh et al. (2007) and Leigh et al. (2011a), but Fiorentino et al. (2014), for instance, used a vertical line in the HRD to delimit the BS region.

Forty-five binaries (4.6\% of all models) fulfill these conditions. Three of them have periods between 0.5 and $1 \mathrm{~d}$, and the rest has periods shorter than $0.5 \mathrm{~d}$. All CBs and NCBs are clustered near the TO point. However, most of them lie below $\mathrm{TO}$, with a mere four falling in the region of BS. The situation is different for binaries with $P>1 \mathrm{~d}$ (not plotted). They all are of Algol-type with low mass ratios and lie in the BS region or redward of it. It is interesting to note that progenitors of CBs and NCBs have initial periods from a narrow interval: 33 out of 45 had periods between 1.7-2.0 d and none had a period longer than $2.4 \mathrm{~d}$. Their total initial masses are between $1.15-1.5 M_{\odot}$, but they are lowered to $1.08-1.37 M_{\odot}$ at the age of 11 Gyr. Short periods dominate among the plotted binaries: apart from three stars with periods longer than $0.5 \mathrm{~d}$ (plus signs), there are only seven more with periods between $0.3-0.5 \mathrm{~d}$ and the rest, that is, 35 stars, has periods shorter than $0.3 \mathrm{~d}$, with the record shortest equal to $\sim 0.14 \mathrm{~d}$. Similarly short periods dominate among field CBs, although here the shortest known period is close to $0.2 \mathrm{~d}$ (Rucinski 2007). We can expect periods shorter than $0.2 \mathrm{~d}$ among low-metallicity CBs, however, because their components are more compact than their solar-metallicity counterparts. Binaries with periods shorter than $0.3 \mathrm{~d}$ have a very high mass ratio, between $0.5-0.8$, whereas those with longer periods have lower ratios down to $0.1-0.2$. This trend results from the dependence of the mass ratio past mass exchange on the difference in core helium content between the components. For two chemically uniform ZAMS stars with unequal masses, the mass transfer stops at $q=1$ Kuiper (1941). The difference in the core helium content between a more and less massive component increases in the course of evolution, so that the later mass transfer takes place, the lower the mass ratio. Observations of field CBs with periods shorter than $0.3 \mathrm{~d}$ indicate that most of them belong to least massive CBs with both components still in the early phases of the MS life (Stępień \& Gazeas 2012). Their mass ratios cluster around 0.5 , as opposed to longer period CBs (Rucinski 2010). Most CBs with periods shorter than $0.3 \mathrm{~d}$ stay in contact for less than $1 \mathrm{Gyr}$, and then coalescence occurs as a consequence of the outer Roche lobe overflow. The longer period binaries live longer as CBs or NCBs (up to $2 \mathrm{Gyr}$, see also Tian et al. 2006) before merging as a result of the Darwin instability (Rasio 1995).

In addition to binaries, 44 mergers ( $4.5 \%$ of all models), formed between 10 and $11 \mathrm{Gyr}$ and evolved to the age of $11 \mathrm{Gyr}$, are listed in Table 3 and plotted in Fig. 6 as open circles. The columns give the name of the model, age of merging, merger mass, luminosity, and effective temperature. In addition to 34 stars lying in the BS region, 10 subgiants or giants are also shown for comparison. These are mergers formed shortly after $10 \mathrm{Gyr}$, which already left the MS and now approach the red giant branch. Because the orbital AM of a binary just before merging is far too high to be accommodated in a spin AM of a single star, most of it must be expelled together with some amount of mass in the form of a ring or disk surrounding the freshly formed star (Rasio \& Shapiro 1995). We adopted $0.1 M_{\odot}$ for the expelled mass, based on an indirect argument presented by Stępień \& Kiraga (2013b). This mass ejected to the distance

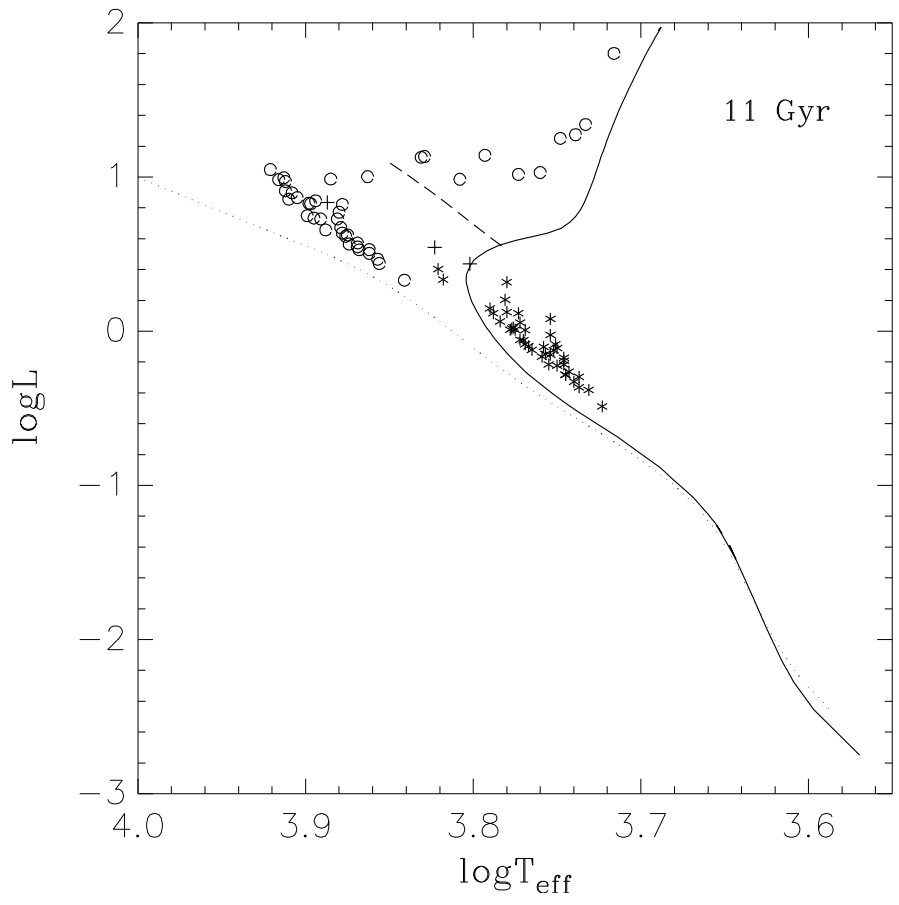

Fig. 6. HR diagram with model CBs, NCBs, and mergers at the age of $11 \mathrm{Gyr}$, together with the corresponding low-metal isochrone. Asterisks denote binaries with an orbital period shorter than $0.5 \mathrm{~d}$, plus signs binaries with periods between 0.5 and $1 \mathrm{~d}$, and open circles denote mergers formed past $10 \mathrm{Gyr}$ and evolved to the age of $11 \mathrm{Gyr}$. The ZAMS is marked with a dotted line and the adopted red boundary of the BS region with long dashes.

of 10-20 $R_{\odot}$ can absorb all excess AM (Sills et al. 2001; Stępień $\&$ Kiraga 2013b). The resulting merger mass is equal to the sum of the component masses minus $0.1 M_{\odot}$. Mergers are assumed to evolve like single stars with the same degree of hydrogen depletion (i.e., no mixing of the merger matter is assumed, see Sills et al. 2009).

Disregarding subgiants, mergers form a well-defined sequence that lies close to the ZAMS for the lowest masses and increasingly deviates from it for the increasing mass. The most massive merger with a mass of $1.43 M_{\odot}$ also has the highest luminosity and already climbs the red giant branch (Fig. 6). All other mergers have masses between $0.98-1.33 M_{\odot}$.

How does the situation change with age? Figure 7 shows similar data as Fig. 6, but 1 Gyr later. Here, again, all binaries with periods shorter than $1 \mathrm{~d}$ at the age of $12 \mathrm{Gyr}$ are plotted with the same coding as in Fig. 6. Most binaries with periods shorter than $0.5 \mathrm{~d}$ are new because almost all those plotted in Fig. 6 did not survive till $12 \mathrm{Gyr}$ and merged in the meantime. But seven binaries from Fig. 6 survived for more than $1 \mathrm{Gyr}$ and are also plotted in Fig. 7. Mergers formed between 11-12 Gyr are shown as open circles. They are supplemented with the mergers from the previous figure, which did not yet climb the red giant branch. Those mergers are shown as filled circles. The numerical data are given in Tables 4 and 5.

The CBs and NCBs present at the age of 12 Gyr have similar properties as those existing $1 \mathrm{Gyr}$ earlier. Almost all short-period binaries lie below or very close to the TO point. Out of 39 binaries (4\% of all modeled), only one lies slightly above TO, but out of 11 long-period binaries 7 lie in the region of BS (two are hidden in a dense clump of filled circles). Similarly as in the previous case, most of the initial orbital periods come from a narrow interval of 1.8-2.1 d that is shifted longward by $0.1 \mathrm{~d}$ compared 


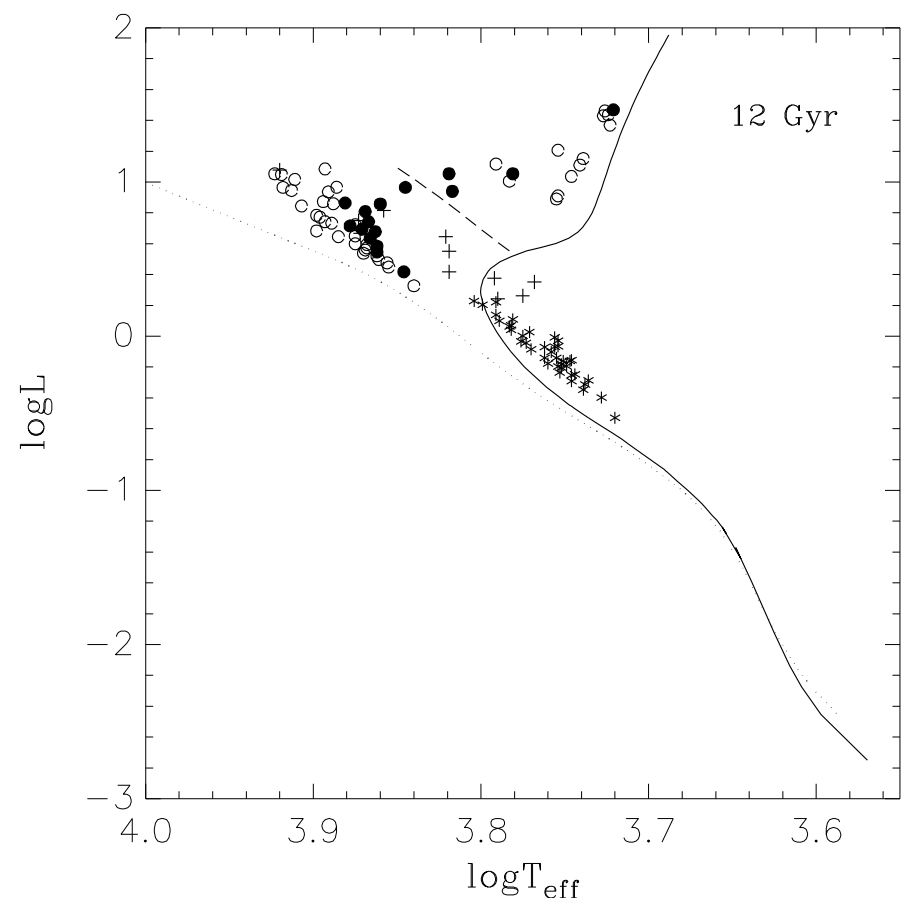

Fig. 7. HR diagram with model CBs, NCBs, and mergers at the age of $12 \mathrm{Gyr}$, together with the corresponding low-metal isochrone. Asterisks denote binaries with an orbital period shorter than $0.5 \mathrm{~d}$, plus signs binaries with periods between 0.5 and $1 \mathrm{~d}$, filled circles denote mergers formed between 10-11 Gyr, and open circles those formed between 11-12 Gyr. All mergers are evolved to the age of 12 Gyr. The ZAMS is marked with a dotted line and the adopted red boundary of the BS region with long dashes.

to the binaries plotted in Fig. 6. The total initial masses lie between $1.1-1.67 M_{\odot}$, but they are lowered to $1.03-1.5 M_{\odot}$ at 12 Gyr.

Of the mergers formed between 11-12 Gyr (open circles in Fig. 7), 30 (3\% of the total number of CCBMs) lie in the BS region and $11(1.1 \%)$ are subgiants. An additional 16 mergers (1.6\%) were formed before $11 \mathrm{Gyr}$ (filled circles). Twelve of these still lie in the BS region, which indicates that a significant fraction of BS spends more than $1 \mathrm{Gyr}$ in this region. Three of them still remain in the BS region even at the age of $13 \mathrm{Gyr}$, that is, almost $3 \mathrm{Gyr}$ after formation (see below).

The situation after another $1 \mathrm{Gyr}$ is displayed in Fig. 8 with the same coding as in Figs. 6, 7. The numerical data of the plotted models are given in Tables 6 and 7 . We have here 46 shortperiod $(4.7 \%)$ and 6 long-period $(0.6 \%)$ CBs and NCBs. Only four binaries (two short-period and two long-period binaries) lie above the $13 \mathrm{Gyr}$ isochrone in the region of BS, and all others lie below the TO point. Similarly as in the earlier age, the initial periods of most of the binaries come from a narrow range 1.9-2.2 d, shifted again longward by $0.1 \mathrm{~d}$, compared to the age of 12 Gyr. The initial total masses of the binaries are between 1.2-1.67 $M_{\odot}$, but the present masses are lowered to $1.0-1.5 M_{\odot}$.

Thirty-six mergers $(3.7 \%)$, formed past $12 \mathrm{Gyr}$, are plotted in Fig. 8 as open circles. They are supplemented with 24 mergers $(2.5 \%)$ formed between 11 and $12 \mathrm{Gyr}$ and 3 more, formed shortly after 10 Gyr. Fifteen mergers fall in the subgiant region, and the rest lies in the BS region, among them the three low-mass binaries mentioned above. Merger masses do not differ from the masses of the mergers plotted in the previous figures: they fall into the interval of $0.93-1.32 M_{\odot}$.

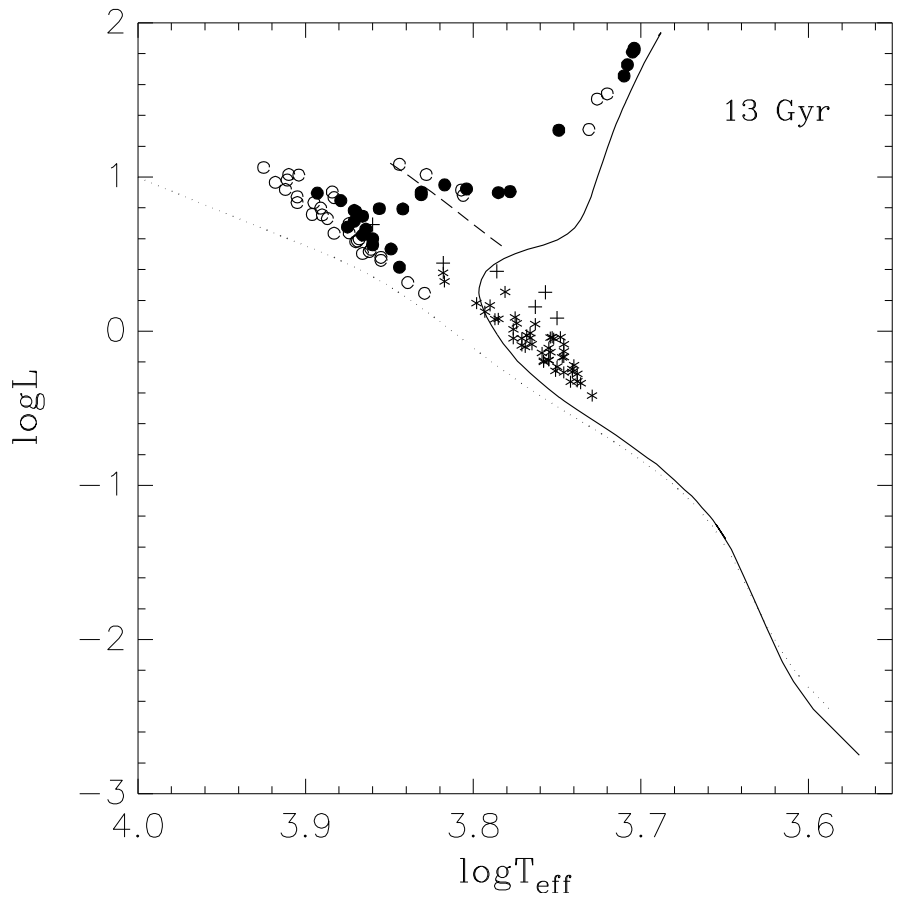

Fig. 8. HR diagram with model CBs, NCBs, and mergers at the age of $13 \mathrm{Gyr}$, together with the corresponding low-metal isochrone. Asterisks denote binaries with an orbital period shorter than $0.5 \mathrm{~d}$, plus signs binaries with periods between 0.5 and $1 \mathrm{~d}$, filled circles denote mergers formed between 10-12 Gyr, and open circles those formed between 12-13 Gyr. All mergers are evolved to the age of 13 Gyr. The ZAMS is marked with a dotted line and the adopted red boundary of the BS region with long dashes.

\section{Comparison of model calculations with observations}

\subsection{Contact and near-contact binaries}

Recently, results of a deep search for photometric variables in a few GCs have been published (Kaluzny \& Thompson 2009; Kaluzny et al. 2010, 2013a,b). In all cases, no CBs were detected among unevolved cluster members, although a prominent binary sequence is visible above the cluster MS on the CMD (see e.g., Kaluzny et al. 2013a). The authors concluded that CBs begin only to appear about 1 mag below the TO. Our results are fully consistent with this conclusion. Assuming that TO lies approximately at $\log L=0$ in Figs. 6-8, the faintest CBs lie about $0.4-0.5$ in $\log L$ below TO, which corresponds to 1-1.2 mag. All model binaries with still lower luminosity are detached. The complete lack of faint, low-mass model CBs results from the adopted value of $1.5 \mathrm{~d}$ for the initial orbital cutoff period. Lowering this value (e.g., to $1 \mathrm{~d}$ or less) leads to a contact configuration reached within the age of a GC by binaries with essentially unevolved, low-mass components. Their absence among the observed CBs is direct evidence that the cutoff period is not shorter than $1.5 \mathrm{~d}$. It may even be close to $2 \mathrm{~d}$ (Stȩpień 2011a). Adopting the latter value would result in a disappearance of several of the faintest CBs in Figs. 6-8 (and some of the plotted mergers).

On the whole, of 975 CCBMs, only about $50(\sim 5 \%)$ are in a contact phase at a given age (more accurately, there are 45,50 , and 52 at the age of 11,12 , and $13 \mathrm{Gyr}$, respectively). Approximately $10 \%$ of these CBs fall in the BS region. Qualitatively similar proportions were obtained by Tian et al. (2006). They modeled over one million binaries, and only less 
than 2000 were in the mass-exchange phase at the age of 4 Gyr, corresponding to the age of M67. About $11 \%$ of these fell in the BS region. A more quantitative comparison between their and our results cannot be made because of distinctly different assumptions adopted.

A lower CB frequency in GCs than in the field has already been noticed by Rucinski (2000). It results most likely from a very low binary frequency of $\sim 0.1$ in GCs (but with a significant scatter among different clusters), compared to 0.5 in the field (Milone et al. 2012), together with a low percentage of binaries in the contact phase at a given age, as shown above. Any statistical comparison of models with observations is therefore extremely uncertain because we have so few observational data. Nevertheless, we can compare the predicted to observed fraction of BSs among all CBs in a GC. We used data for two clusters observed by Kaluzny and his collaborators with the highest number of CBs (except for $\omega$ Centauri). In M4, Kaluzny et al. (2013a) detected nine CBs with one BS among them, and in 47 Tuc, Kaluzny et al. (2013b) identified 15 CBs or NCBs, of which six are BS. The resulting fractions are 0.1 and 0.4 , respectively. We did not take into account $\omega$ Cen with the richest population of CBs (Kaluzny et al. 2004) because it is highly atypical and the accurate number of member CBs is not well known, although the approximate data indicate a similarly high ratio as in case of 47 Tuc. The predicted fractions range from 0.08 to 0.2 for the three considered cluster ages. As we see, they are close to the lower observed value but are at odds with the higher value. This may indicate some deficiencies of the CCBM, for instance, too short initial cut-off period, a too high AML rate at short periods, or a too low mass-transfer rate. With the lower AML rate and/or higher mass transfer rate, a binary stays longer in contact and can reach a lower mass ratio before merging. Many of the short-period CBs have a rather high mass ratio of about $0.7-0.8$ at the time of merging, whereas field CBs with $P \lessgtr 0.3 \mathrm{~d}$ center around a value of 0.5 (Rucinski 2010; Stępień \& Gazeas 2012). The lower mass ratio means more mass transferred to the gainer, hence its higher mass and higher position on the HRD, that is, a higher probability for entering the BS region. There may also still be another explanation of the discrepancy: strong fluctuations of this fraction among different clusters suggest a nonuniform BSs formation rate in some clusters, with individual bursts occurring in the recent past (see below). Regardless of the reason, it is apparent that profound differences occur among GCs, which makes a comparison of theoretical predictions with individual clusters uncertain.

A direct comparison of CCBMs to the observed parameters of individual CBs or NCBs is hampered because only very few high-quality data are available for these GC members. To our knowledge, V60, an Algol-type BS in M55, is the only NCB with parameters based on light and velocity curves (Rozyczka et al. 2013). Generally, to find a progenitor for a given binary, a fine-tuning of the initial parameters is needed. Here we do not attempt it, but we can still find a model that reproduces V60 quite well. This is the most massive CCBM, that is, $0.89+0.8(1.9)$. Its parameters at the age of about $11 \mathrm{Gyr}$ are compared with the observed values for V60 in Table 8 . The positions of the two binaries are also shown in the HRD (Fig. 9), where a diamond corresponds to V60 and the plus sign to the model. Another model, $0.9+0.8(1.9)$, evolves in about $11 \mathrm{Gyr}$ to a similar configuration with a period of $1.18 \mathrm{~d}$ and component masses of 0.24 and $1.30 M_{\odot}$, which is also not far from V60, but its luminosity, $\log L=0.998$, is somewhat too high.

As we see, the model parameters agree with the observed values within about $2 \sigma$, even though the model is not sufficiently massive to better reproduce the observations. The total mass of V60 is equal to $1.59 M_{\odot}$, compared to only $1.54 M_{\odot}$ for the model. A slight mass increase, together with a correct period and metallicity should give a better agreement. When the initial parameter values of CCBM were specified, the following evolution was fully determined - we did not introduce any free parameters that would, for instance, describe additional mass and/or AM loss.

The mass and radius of the less massive component of V60 indicate that it is an evolved star with a helium core of $\sim 0.15 M_{\odot}$, whereas its companion is a ZAMS star. The binary is thus a typical short-period Algol. It is commonly accepted that such a configuration results from a rapid mass transfer following a RLOF by an evolved primary. In effect, a binary is formed showing the well-known Algol paradox. No other way of producing an Algol-type binary seems to be viable, although many details of evolution preceding RLOF, starting from the origin of the initial binary, possible dynamical interactions with other stars, or amount of mass and AM lost during the consecutive evolutionary phases, are still unknown and need to be assumed for the calculated model. Even if all these details are fixed, as in case of the CCBM code, the results are equivocal. Different combinations of the initial parameters may result in the same final values, except that they have different age (Stępień 2011b; Pietrzyński et al. 2012). If the age of the modeled binary is not known, ambiguity remains. For a binary member of a GC the age is known, which additionally constrains a possible initial model.

Stellar parameters have also been obtained for a few more CBs and NCBs in GCs, based solely on the photometric observations. These are NH 19, 30 and 31 in NGC 5466 (Kallrath et al. 1992), NJL 5 and V239 in $\omega$ Cen (Helt et al. 1993; Li \& Qian 2012), V1 in NGC 6397 (Rubenstein \& Bailyn 1996), V228 in 47 Tuc (Kaluzny et al. 2007; Sarna 2008), and V47 and V53 in M4 (Liu et al. 2011). In most cases, however, the resulting binary parameters are very uncertain, so any fine-tuning of the initial parameters to reproduce the obtained results must await better observations. Still, if the data are acceptable, we can always find a model close to the observed variable. Li \& Qian (2012) analyzed the photometry of V239, an Algol-type binary with $P=1.19 \mathrm{~d}$, whose light curves were obtained earlier by Kaluzny et al. (2004). The solution indicates that the orbit inclination is close to $90^{\circ}$, hence the resulting parameters are probably almost correct. The resulting component masses are $M_{1}=0.07$ and $M_{2}=1.20 M_{\odot}$. The model $0.8675+0.6(2.0)$ evolves after 13.5 Gyr into a binary with a period of $1.36 \mathrm{~d}$ and component masses $0.10+1.20 M_{\odot}$. We stopped the calculations at this age, but the mass transfer was still ongoing and the period was decreasing, so after the next few time steps the binary would resemble V239 even more. V228 in 47 Tuc is another Algol-type star. Its present parameters are $P=1.15 \mathrm{~d}$, $M_{1}=0.20$ and $M_{2}=1.51 M_{\odot}$ (Kaluzny et al. 2007). Sarna (2008) modeled this star using his evolutionary program. The program did not include magnetized winds but, instead, it contained two free parameters individually adjusted and describing the total mass and AM lost during the evolution. He concluded that the progenitor binary had a period of $1.35 \mathrm{~d}$ and component masses equal to 0.88 and $0.85 M_{\odot}$. Allowing for mass loss by the winds and the present total mass of V228, we estimate that the initial component masses must have been close to $0.9 M_{\odot}$. None of the CCBMs is that massive. The metallicity of 47 Tuc is also several times higher than used in CCBMs. However, the model $0.96+0.93(2.3)$ with the solar composition, calculated for another purpose some time ago, evolves after 12.4 Gyr into the configuration with period of $1.13 \mathrm{~d}$ and component masses 
Table 8. Comparison of the observed parameters of binary V60 from M55 with one of CCBMs.

\begin{tabular}{lllllllll}
\hline \hline Parameter & $P(\mathrm{~d})$ & $a\left(R_{\odot}\right)$ & $M_{1}\left(M_{\odot}\right)$ & $M_{2}\left(M_{\odot}\right)$ & $R_{1}\left(R_{\odot}\right)$ & $R_{2}\left(R_{\odot}\right)$ & $\log \left(L / L_{\odot}\right)$ & $\log T_{\mathrm{e}}$ \\
\hline V60 & 1.18 & $5.49 \pm .05$ & $0.33 \pm .02$ & $1.26 \pm .03$ & $1.48 \pm .01$ & $1.10 \pm .02$ & $0.813 \pm .013$ & $3.832 \pm .008$ \\
Model 0.89+0.8(1.9) & 1.18 & 5.41 & 0.28 & 1.26 & 1.42 & 1.13 & 0.880 & 3.854 \\
\hline
\end{tabular}

$0.20+1.49 M_{\odot}$. This is quite close to the observed parameters of V228.

V47 and V53 are CBs with short periods of 0.27 and $0.31 \mathrm{~d}$. The photometric solutions resulted in component masses of $0.20+1.66 M_{\odot}$ for V47 and $0.74+0.91 M_{\odot}$ for V53 (Liu et al. 2011). Unfortunately, the orbit inclination resulting from the photometric solution is equal to about $72^{\circ}$ and $40^{\circ}$ for $\mathrm{V} 47$ and V53, respectively, so quite far from $90^{\circ}$. In such cases the mass ratio is poorly constrained and can be far from the spectroscopic value (Rucinski 2006). The binary V47, with a total mass of $1.86 M_{\odot}$, is substantially more massive than any of the CCBMs discussed in this paper. In fact, the mass of the primary is higher than twice the TO mass (equal to $0.8 M_{\odot}$, Kaluzny et al. 2013a), which casts some doubt on its determination, although another route of producing a massive merger is possible, involving multiple mergers or collisions (van den Berg et al. 2001; Sandquist et al. 2003; Leigh \& Sills 2011). Clearly, a high mass of the primary is also difficult to reconcile with its low luminosity $L_{\mathrm{p} r}=2 L_{\odot}$ (Liu et al. 2011). The expected initial mass of another binary, V53, is about $1.8 M_{\odot}$, which is again beyond the limits considered by us. Still, we can find models in the CCBM set with the period and $q$ similar to V53, for instance, the model 0.8675+0.8(1.9), which evolves after $11 \mathrm{Gyr}$ into a binary with a period of $0.305 \mathrm{~d}$ and component masses of $0.66+0.86 M_{\odot}$. We did not attempt to find possible progenitors of the binaries investigated by Kallrath et al. (1992), Helt et al. (1993), and Rubenstein \& Bailyn (1996) because their parameters are even more uncertain.

A more accurate search for progenitors of individual binaries in GCs will be the subject of a separate paper.

\subsection{Blue stragglers}

Recent progress in understanding the origin and properties of BS in GCs has been enormous, mostly due to observations of GC cores with the Hubble telescope of Piotto et al. (2004), Leigh et al. (2007), Beccari et al. (2012), Dalessandro et al. (2013a, b), and Sanna et al. (2014). A comprehensive review of observations and resulting conclusions can be found in Boffin et al. (2015).

To compare models with observations, we first need to specify which members of a GC are classified as BS. There is no single generally accepted method. To avoid contamination from photometric errors of MS stars at the TO point and blends (particularly in a cluster core), selection criteria require that a BS candidate be brighter than TO by a predefined quantity. In addition, a BS should lie at the extension of the cluster MS, but the width of the BS band is not strictly determined and varies among different authors. Some authors plot boxes in the color-magnitude diagram (CMD), see, for instance, Leigh et al. (2011a), Dalessandro et al. (2013b), Li et al. (2013), and Fiorentino et al. (2014), others select BS by analyzing individual candidates and applying specific criteria (Moretti et al. 2008; Ferraro et al. 2009). The resulting set of BS approximately extends over 1-2 mag in $V$ and $0.4-0.5$ in $B-V$. Most of the observed BSs lie along the extension of the MS, but some stars, lying already beyond the TAMS, where evolved single stars and binaries can be found, are often included in the BS samples. The BS region adopted in this paper is bound by the ZAMS, the corresponding isochrone $(11,12$, or $13 \mathrm{Gyr})$, and the ZAMS line shifted by $\Delta L=+0.8$ (Figs. 6-8).

Ferraro et al. (2009) detected a gap dividing BS in the cluster M30 into two sequences: blue and red. They argued that the separation results from two different formation mechanisms: the blue sequence, lying close to the ZAMS, originated from collision-formed mergers, whereas the red sequence that contains BS formed through mass transfer in binaries. Similar sequences, albeit with a less pronounced gap between them, were detected in NGC 362 (Dalessandro et al. 2013b). The blue sequence agrees with the expected position of mergers formed by a collision of two stars with masses close to half a TO mass. Such stars are hardly evolved before merging, hence a freshly formed merger is expected to lie close to ZAMS. The line separating both sequences was adopted after Tian et al. (2006). It lies approximately at the extension of the MS binary sequence, that is, the ZAMS line shifted upward by $0.75 \mathrm{mag}$, and reproduces the expected position of unevolved, equal-mass binaries. It follows from this interpretation that the blue sequence consists of single unevolved stars, whereas the red sequence should consist of binaries. In addition, the distribution of BS with a clear gap between both sequences requires that they were all formed in a single recent burst, identified by Ferraro et al. (2009) with a cluster core collapse. The subsequent evolution of blue BSs will smear the distribution over CMD, so the gap should disappear. In addition, if BSs are formed continuously in a cluster, none of the features observed by Ferraro et al. (2009) and Dalessandro et al. (2013b) should occur ( $\mathrm{Li}$ et al. 2013). To see how the models are situated relative to the blue and red sequences seen in M30 and NGC 362, we plot all mergers from Tables 3, 5, and 7 as filled circles in Fig. 9 and all binary BSs as asterisks. A few binaries with $1<P<3 \mathrm{~d}$ are also added. We do not list numerical data for them because the accuracy of the CCBM calculations decreases with increasing period of an evolutionary advanced binary, therefore their parameters are rather uncertain. The isochrones at 11, 12 and 13 Gyr are also plotted as solid lines. In addition, the ZAMS line (dotted), the upper edge of the binary BS region, determined by Tian et al. (2006) (broken line), and the adopted red boundary of the BS region (long dashes) are shown. The broken line delimits the region of binary BSs from the blue side. All CCBMs but one lie to the right of this line. The exception is model $0.8675+0.6(1.9)$. It became a $\mathrm{CB}$ at the age of $11 \mathrm{Gyr}$, but was lying below TO at that time (Table 2). By a continuing mass transfer, the gainer reached a mass of $1.161 M_{\odot}$ at $12 \mathrm{Gyr}$ and the binary moved to the blue part of the BS region (Table 4). The gainer is hardly evolved at that age and the influence of the redder secondary is insufficient to move the binary to the red part of the BS region. This indicates that in exceptional cases CBs can also be found in the blue sequence, as is observed in M30 and NGC 362 (Ferraro et al. 2009; Dalessandro et al. 2013b).

In contrast to binaries, the model mergers can be found in both parts of the BS region (Fig. 9). Those with the lowest masses, around $1 M_{\odot}$, lie close to ZAMS, along a narrow blue 


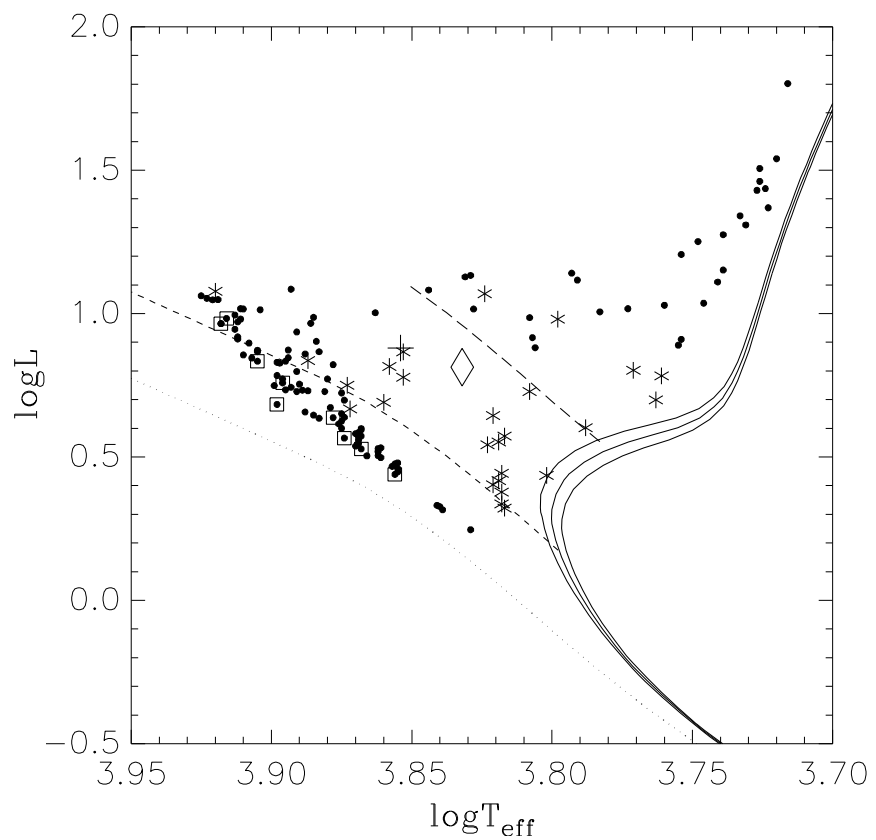

Fig. 9. Collective HR diagram of all mergers (filled circles) and all binary BS (asterisks) from Figs. 6-8, together with the corresponding isochrones. Rapidly rotating mergers are additionally marked with squares. The position of the eclipsing binary V60 from M55 and of the model $0.89+0.8(1.9)$ are plotted as a diamond and a plus sign. The ZAMS and the blue boundary of the binary BS region determined by Tian et al. (2006) are shown as a dotted and broken line. Long dashes mark the adopted red boundary of the BS region.

sequence. They were formed by two unevolved stars, hence resulting mergers are also unevolved even at the age of $13 \mathrm{Gyr}$. Their masses are between $0.9-1.1 M_{\odot}$. A gap separating them from the binaries is clearly visible. However, mergers more massive than about $1.1 M_{\odot}$ are formed by two stars of which at least one has substantially depleted core hydrogen, which means that they are evolutionary advanced already immediately after formation. In effect, they arrive to the sequence tilted to ZAMS and extending to the red part of the BS region. Similarly, mergers formed collisionally in the evolutionary models of a GC are scattered over the whole BS region (Sills et al. 2009, 2013; Chatterjee et al. 2013). We conclude that binaries that move to the $\mathrm{BS}$ region as a result of mass exchange, arrive predominantly to the red of the line determined by Tian et al. (2006). However, the existence of a gap delimiting the narrow sequence of BSs close to ZAMS and extending to the most massive BSs cannot be reconciled with a continuous merger production. A burst in their production seems to be the most plausible explanation, as suggested by Ferraro et al. (2009), although this picture per se is not proof of the collisional formation of blue BSs.

Masses of a few binary BS are known from the solution of the eclipsing light curves, sometimes supplemented with radial velocity curves (see previous subsection). Less is known about masses of single BS. Until recently, two methods have been applied to determine their masses. The first was based on spectral and photometric observations, the second on the evolutionary tracks in the CMD (Shara et al. 1997; Geller \& Mathieu 2011, 2012; De Marco et al. 2005; Kaluzny et al. 2007, 2009, 2013a). Unfortunately, discrepancies often occur when both methods are used on the same object (De Marco et al. 2005; Geller \& Mathieu 2012). Recently, a new method was introduced by Fiorentino et al. (2014). It is based on the comparison of the pulsation properties of BS that belong to SX Phe - type variables. Applying

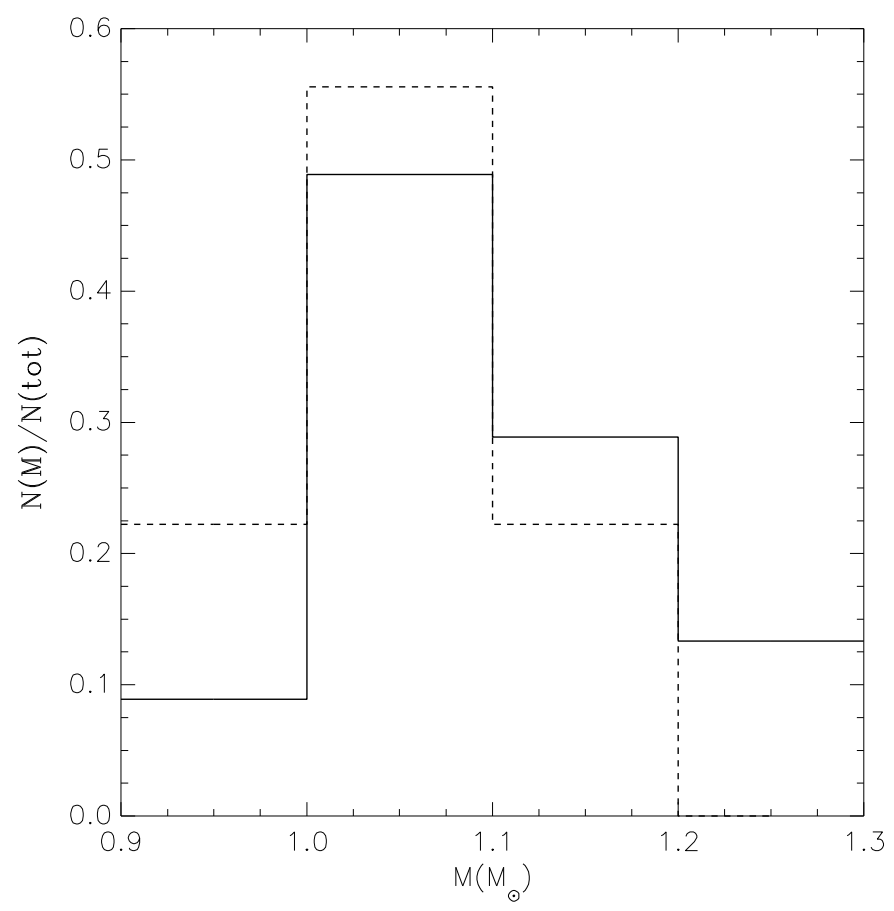

Fig. 10. Mass distribution of the BS mergers from Table 7 (solid line) compared to the observed distribution of the pulsation masses of SX Phe stars in NGC 6541 (broken line). Both distributions are normalized to unity.

this method to SX Phe stars in NGC 6541 resulted in the pulsation masses of nine variable BSs. Six of them are pulsating in the fundamental mode and three in the first overtone. For the last three stars the authors also calculated masses assuming fundamental pulsation, but they clearly preferred the first overtone identification. The masses agree very well with evolutionary masses, particularly when they are binned according to luminosity. We compare them with masses of the mergers from the BS region in Fig. 8. There are 45 such mergers in Table 7. Figure 10 shows the mass distribution of the model mergers (solid line) together with the observed distribution (broken line). Both distributions are normalized to unity. The agreement is surprisingly good, taking into account the low number of the observed masses and the fact that SX Phe instability strip is narrower than the BS region. The lack of the observed masses in the last bin, between $1.2-1.3 M_{\odot}$, most likely results from this restriction.

Recently, spectroscopic studies of BSs in a number of GCs have been performed with the aim to investigate the mechanisms of their formation (Lovisi et al. 2013; Lovisi 2014, and references therein). One of the goals was to measure rotation rates of BSs. Freshly formed binary mergers are expected to rotate rapidly even though a great majority of the orbital AM is expelled with some matter (Rasio \& Shapiro 1995). Rapid rotation is also expected for collision-formed mergers unless the collision is strictly central (Sills et al. 1997, 2001). But cool stars lose AM by the magnetized wind. In effect, they spin down from an initial rotation period of a fraction of a day to about one week in $10^{8}$ years, that is, in terms of the equatorial velocity, from $v_{\mathrm{eq}} \approx 100-150 \mathrm{~km} \mathrm{~s}^{-1}$ to less than $10 \mathrm{~km} \mathrm{~s}^{-1}$ (Barnes 2009). Except, possibly, for the most massive mergers, all other BSs in GCs are magnetically active and spin down on a timescale of $10^{8}$ years. In Fig. 9 we highlighted all mergers formed in the last $10^{8}$ years before 11,12 , and $13 \mathrm{Gyr}$ with squares. Rotation evolution is not included in the CCBM code, but we assume that freshly formed mergers rotate rapidly. As we see, all these 
mergers lie close to the high-temperature boundary of the BS region, indicating that they are still unevolved. There are only nine rapid rotators in the figure, that is, about $10 \%$ of all BS mergers. This is an upper limit for the expected fraction of rapid rotators in a GC, achieved only if mergers formed in the last Gyr exist in the BS region. All mergers formed earlier will add to slowly rotating, thus decreasing the fraction of rapid rotators. For example, only three such rotators are expected to occur among 48 BS mergers in Fig. 8 (see Table 7), which is equivalent to about $6 \%$.

So far, accurate observations of $v \sin i$ of several BSs in four GCs have been obtained: 47 Tuc (Ferraro et al. 2006), M4 (Lovisi et al. 2010), NGC 6397 (Lovisi et al. 2012), and M30 (Lovisi et al. 2013). The total number of BSs with a measured rotation rate in these clusters is 96 , ten of which are rapid rotators with $v \sin i>50 \mathrm{~km} \mathrm{~s}^{-1}$ (we exclude one W UMa-type binary counted as a rapid rotator in M30), this is about $10 \%$. This is close to the predicted value (see above). Moreover, all rapid rotators in M4 and moderate rotators (with $v \sin i>10 \mathrm{~km} \mathrm{~s}^{-1}$ ) in 47 Tuc lie near the blue boundary of the BS region, indicating that they are freshly formed (Ferraro et al. 2006; Kaluzny et al. 2013a). However, this statistics is poorer when individual clusters are considered: eight rapid rotators are present in M4 (40\% of the total measured sample in this cluster), whereas only two are members of three other clusters, of more than 80 stars measured. This shows again that GCs are very different from each other, and a comparison of an individual cluster with theoretical predictions may be misleading.

If mergers spin down as a result of magnetized winds, we expect a gap in the rotation distribution between rapidly and slowly rotating stars, resulting from the existence of the socalled Vaughan-Preston gap (Barnes 2011). The rotation distributions of BSs show a gap (see Fig. 10 in Lovisi et al. 2012), although its statistical significance is low.

Another goal of the spectroscopic survey of BSs was to search for anomalous surface abundances in BSs that would indicate the material processed by the CNO cycle (Ferraro et al. 2006; Lovisi et al. 2010, 2012, 2013). As a star evolves, the abundance of carbon ${ }^{12} \mathrm{C}$ and ${ }^{16} \mathrm{O}$ decreases, whereas the abundance of nitrogen ${ }^{14} \mathrm{~N}$ increases in the layers where nuclear reactions operate (Iben 1967). The transition between the cosmic and modified abundance moves with time slowly outward until it reaches the mass fraction $\sim 0.3$ for solar-type stars (Iben 1967). If enough matter is transferred to the companion, the material processed by nuclear reactions is exposed, hence anomalous abundances of these elements are expected to be present on the surfaces of both stars. Detection of any of these anomalies in a BS can be treated as an evidence for the mass transfer channel of its formation. An underabundance of carbon has indeed been observed in field Algols (Sarna \& De Greve 1996).

It follows from these considerations that at least $70 \%$ of the loser mass needs to be transferred to its companion to expose altered abundances (Iben 1967; Sarna \& De Greve 1996). In most CCBMs, only about $40-50 \%$ of the mass is transferred to the gainer before the two components merge. We should therefore not expect to see any CNO peculiarities in these binaries and mergers. A sufficient mass fraction is transferred only in a few binaries that ultimately merge as a result of the Darwin instability. There are about ten such BSs among the binaries plotted in Fig. 9. All except one lie in the red part of the BS region or in the subgiant region. To these, one merger resulting from the model $0.8675+0.4(2.3)$ can be added because it was formed by the Darwin instability just before 13 Gyr. Its surface layers may also show carbon and oxygen depletion. Altogether, less than
$10 \%$ of the plotted BSs are expected to show an underabundance of carbon and oxygen, which means that the probability of finding such an object among a random sample of BSs does not exceed 0.1 .

The observations of GCs agree with these expectations. They are summarized by Ferraro et al. (2015) and Lovisi (2014). C and O depletion for a few BSs in 47 Tuc, M30 and $\omega$ Cen was detected, but none of the observed BSs in three other clusters showed the expected alterations. The authors estimated that only about $10 \%$ of BSs may show CO depletion. That was fewer than they expected, therefore they speculated that the low percentage may result from the transient character of the alterations. While this assumption may still be viable, it is not needed to explain the observations.

\section{Discussion and conclusions}

We computed an extended set of CCBMs with initial parameters expected to reproduce observations of $\mathrm{CBs}$, NCBs, and BSs in GCs. The initial component masses were between $0.7-0.9 M_{\odot}$ for the primary and $0.4-0.8 M_{\odot}$ for the secondary components. The initial orbital period distribution had a short-period cut-off at $1.5 \mathrm{~d}$ resulting from the accepted binary formation mechanism (Boss 1993; Bonnell 1994; Kratter et al. 2010; Machida et al. 2008) and a maximum between $2-3$ d resulting from the KCTF mechanism operating on suitably shaped triples (Eggleton \& Kiseleva-Eggleton 2006; Perets \& Fabrycky 2009). The metal abundance was fixed at $Z=0.001$. Magnetized winds from both components were adopted as the driving force for orbit evolution. Evolutionary calculations were carried out to the age of 13.5 Gyr, but detailed analysis of the evolved objects was performed for three ages: 11, 12, and $13 \mathrm{Gyr}$.

The results show that all binaries with primary masses lower than $0.7 M_{\odot}$ and almost all with masses $0.75 M_{\odot}$ remain detached untill the age of 13.5 Gyr. On the other hand, almost all binaries with initial primary masses close or equal to $0.9 M_{\odot}$ complete the evolution as mergers or common-envelope products before they reach the age of 11 Gyr. Only binaries with primary masses embracing the TO mass at a specified age of 11,12 or $13 \mathrm{Gyr}$ and with initial periods from a narrow interval around $2 \mathrm{~d}$ form CBs or NCBs with periods shorter than $1 \mathrm{~d}$, which should be observed as W UMa-type stars in a GC of that age. Most of these variables do not survive for more than 1 Gyr and either form a merger or an Algol-type star with a period of several days. Consequently, only $4-5 \%$ of the modeled binaries are in contact or near-contact at each specified age. Observations show, indeed, that W UMa-type binaries are rare among GC members (Rucinski 2000). Moreover, about $90 \%$ of model CBs lie close to, but below TO point on the HRD, which means that on average, only one in ten CBs falls in the BS region. Considering the faint limit for $\mathrm{CBs}$, the absence of model CBs fainter than $\sim 1$ mag below TO point agrees well with observations of GCs (Kaluzny \& Thompson 2009; Kaluzny et al. 2010, 2013a).

As the calculations show, freshly formed mergers have masses between $0.9-1.5 M_{\odot}$, as compared to a typical TO mass of $\sim 0.8 M_{\odot}$ in a GC. Thus, all they arrive in the BS region. In effect, they outnumber binary BSs by at least an order of magnitude, so among the random sample of BSs, fewer than $10 \%$ are expected to be close binaries. The observations support this result, although substantial fluctuations of this fraction occur among individual clusters (Ferraro et al. 2009; Dalessandro et al. 2013b; Kaluzny et al. 2010, 2013a). Algols with periods longer than $1 \mathrm{~d}$ occur predominantly to the red of the BS region because 
of the influence of a cool, Roche-lobe filling component that reddens the binary.

All mergers with masses lower than $1.1 M_{\odot}$ are still unevolved and lie along ZAMS, thus forming a blue BS sequence. On the other hand, all binary BSs lie in the red part of the BS region, thus forming a red sequence. A gap separates the two sequences. The existence of such a gap has been noted in two GCs, but it was linked to a highly nonuniform BS formation rate (Ferraro et al. 2009; Dalessandro et al. 2013b). Further investigations should resolve this discrepancy.

The uncertainties of the CCBMs mostly stem from the uncertainty of the coefficients in Eqs. (7), (8) and the accuracy of the stellar models. An increase of the AML rate by $30 \%$ results in a decrease of the orbital period at RLOF by about $0.2-0.3 \mathrm{~d}$. This has a different influence on binaries with RLOF occurring on the MS and occurring beyond the TAMS. In the former, RLOF takes place earlier, when the primary is less evolved, hence the mass ratio past rapid mass exchange is higher by about 0.05-0.1. Component merging and its following evolution also occur earlier. In effect, CBs observed at the specified age originate from binaries with initial periods longer by the same amount. In the latter, the shorter period at RLOF has a negligible effect on the process of mass exchange because the fast radius increase of the subgiant component changes the time of RLOF by a mere few time steps. The mass ratio past rapid mass exchange also changes little, so only the orbital period is shorter, again, by the same amount. A decrease of the AML rate by $30 \%$ has the opposite effect to the same extent on the binary parameters. In short, the uncertainty in the AML rate of $30 \%$ translates into an uncertainty of about $10 \%$ in the binary and merger parameters.

A decrease of mass-loss rate by a factor of two has an even weaker effect. The total mass lost of a CB is between $6-7 \%$ of the initial binary mass. Its cut in half shortens stellar evolutionary time scales by about $10 \%$ so RLOF occurs somewhat earlier, but the final parameters of the considered models agree within $4-5 \%$ with those presented above. An increase of the massloss rate by a factor of two influences the resulting parameters stronger, but, again, the change is lower than $10 \%$. Only a substantially higher mass-loss rate (e.g., by a factor of 5) would qualitatively change the developed picture. Spherically symmetric mass loss always causes a period increase. For each AML rate there exists a maximum value of the mass-loss rate such that for higher mass-loss rates orbit widens. That would substantially change the binary evolution. There are no accurate measurements of mass-loss rates for low-mass stars, therefore an extrapolation of data for solar-type stars was used. The mass-loss rates from Eq. (7) give between $0.5-1.5 \times 10^{-11} M_{\odot}$ per year for a $0.8 M_{\odot}$ star at the ZAMS and at the TAMS, respectively, and $2-7 \times 10^{-12} M_{\odot}$ per year for a $0.5 M_{\odot}$ star. Other authors suggested similar rates for low-mass dwarfs, for example, Cranmer $\&$ Saar (2011), or even somewhat lower $\left(\sim 10^{-12} M_{\odot}\right.$ per year, van den Oord \& Doyle 1997). It seems therefore that a substantially higher mass-loss rate is unlikely.

An assessment of the uncertainties connected with stellar evolutionary models is more difficult. Even the models of single stars differ significantly among different authors because of different input physics (Verbunt \& Phinney 1995; Nataf et al. 2012). In particular, the timescales of the consecutive evolutionary phases and radii of the models may differ by several percent. Of the same order is the uncertainty in stellar radii, resulting from magnetic inflation observed in rapidly rotating cool dwarfs (Torres et al. 2010). Fortunately, this uncertainty has only a minor effect on the final results. The age of RLOF and the merging time change by several time steps when we change the stellar radius by $10 \%$, and the period boundary between CBs and NCBs (which, anyway, we treat together) shifts only little.

These considerations about binary BSs only relate to close binaries. Any BS can also be a member of a long-period binary. In fact, our assumptions require that most of these objects possess (or possessed in the early evolutionary phases) a tertiary component that shortens the inner binary period by the KCTF mechanism, as suggested by Perets \& Fabrycky (2009). These authors also discussed the observational consequences of this assumption. They compared the timescales for dynamical disruption of triples in cores and outer regions of stellar clusters with the timescale for a formation of a close binary. Both time scales are functions of the outer binary period. It follows from the comparison that for $P_{\text {out }} \leq 10^{4} \mathrm{~d}$ dynamical encounters are ineffective in the outer parts of a GC. Thus a large part of BSs should occur there in triples (before merging) or in binaries. Only those formed in initially soft triples might be disrupted. Altogether, the fraction of binaries and triples among BS should be higher than the overall binary fraction in the environment where they are observed, and most should be hard. The much higher frequency of the dynamical interactions in GC cores make the prediction more difficult. The timescale for the close binary production is short enough for $P_{\text {out }} \leq 10^{4} \mathrm{~d}$, so the KCTF mechanism can still operate in a GC core, but a high interaction rate results in a disruption, a change of the configuration or a merger of the inner binary. The outer binaries are even more susceptible to disruption or component exchange. In effect, the fraction of binary BSs in a GC core is expected to be lower and they will have different parameter distribution from those in low-density regions. If the triple system is not disrupted, a second-generation $\mathrm{BS}$ may be formed when a first-generation BS, considered here, transfers matter to the original tertiary after reaching the red, or asymptotic giant branch. In effect, a population of BSs with white dwarf companions is expected. The observations of the old open cluster NGC 188 revealed that a significant fraction of BSs has such companions (Gosnell et al. 2014). Unfortunately, no such observations for GCs are available.

For very few individual CBs in GCs, sufficiently accurate parameters are known. Although we did not attempt at present to find progenitors that fully match the observations, we can always find a model within the CCBM set that closely reproduces the observed parameters. For example, the model with initial masses $0.89+0.8 M_{\odot}$ and the initial orbital period of $1.9 \mathrm{~d}$ evolves after $11 \mathrm{Gyr}$ into a binary with masses $1.26+0.28 M_{\odot}$ and a period of $1.18 \mathrm{~d}$, which matches the variable V60 from M4 very well, for which the most accurate data have been determined (see Table 8 and Fig. 9). Adding age and metallicity as additional constraints should result in a unique initial model for this binary.

One of our main results shows that the binary-originated BS cover most of the region on the HRD where the observed BSs occur. The least massive mergers lie close to ZAMS. This is due to the low masses of the merging components with hardly depleted core hydrogen. The product of merging is then unevolved and can remain in the BS region for 2-3 Gyr before moving to the red giant region. They are indistinguishable in the HRD from the collision-originated mergers of two low-mass stars. The higher the component masses, the more advanced evolutionally the merger, hence the blue boundary of the BS sequence turns more and more away from ZAMS (see Figs. 6-8). Evolutionary advanced mergers with core hydrogen depleted and binaries past RLOF populate the red part of the BS region. Only about 6-10\% of the mergers are expected to rotate rapidly, and all probably lie close to the blue BS boundary. Similarly, a low fraction of BSs may show chemical alterations resulting from exposing layers 
where the CNO cycle operated. They should occur predominantly among binaries with low mass ratios where a sufficiently large amount of mass has been transferred between the components. These predictions agree with observations of a few GCs (Ferraro et al. 2006; Lovisi et al. 2010, 2012, 2013).

To conclude, none of the observed parameters of an individual single BSs can uniquely point to its origin: binary or collisional. The presence of binaries among BSs obviously indicates that at least a fraction of these objects has a binary origin. Can this be true for most BSs? We noted profound differences among individual GCs regarding the properties of BSs, therefore a single picture that would be applicable for all clusters seems improbable. Evolutionary modeling of a GC, including stellar and binary evolution, should provide credible predictions about the relative importance of different production channels (Hypki \& Giersz 2013; Chatterjee et al. 2013). On the other hand, a statistical analysis of GCs should help to verify these predictions (Milone et al. 2012; Sills et al. 2013). It seems at present that models of GCs favor formation mechanisms involving binaries. Hypki \& Giersz (2013) modeled a cluster with $10^{5}$ objects, $20 \%$ of which are binaries. They considered ten different ways of BS formation. The results showed that single-star collisions produce a negligible number of BS, $13.6 \%$ are formed by collisions involving binaries (single-binary and binary-binary), and the rest by binary mergers. They noted, however, that these results may not apply to more massive and denser clusters.

Somewhat different results were obtained by Chatterjee et al. (2013), who showed that in a dense environment (above $10^{3} M_{\odot} / \mathrm{pc}^{3}$ ) most of the BS are formed by collisions mediated by binaries, but in lower-density clusters most BS (up to 60\%) result from mass transfer in binaries. In effect, we can expect a varying proportion between both kinds of BSs among different clusters. This is in line with Ferraro et al. (2012), who argued that BSs formed in cluster cores originate from collisions, whereas those in cluster peripheries result from binary evolution. Therefore a flat density distribution is seen in dynamically young clusters, whereas an increasing concentration toward the cluster center is visible in dynamically older clusters when more massive BSs from the outer parts gradually migrate into the core. In addition, Li et al. (2013) announced the observations of bimodal distribution of BSs in the Large Magellanic Cloud GC Hodge 11. They noted that BSs from the central distribution peak lie within the blue sequence in the HRD, whereas those from the outer peak are placed in the red part of the BS region. Assuming that the position in the HRD uniquely determines an origin of a BS, they concluded that core BSs are collisionally formed, whereas binary-formed BSs dominate in the outer parts of the cluster. The results of the present paper show, however, that this assumption is not correct. We also note that the statistical comparison of the BS population in several GCs with the simplified formation model clearly indicates that binary-formed BSs dominate those collisionally formed everywhere (Leigh et al. 2011b).

In brief, the present evolutionary model of cool close binaries fully explains the origin, evolutionary stage, statistical properties, and individual physical parameters of CBs and NCBs observed in GCs.

Our results favor a binary origin of BSs, but this is still an open question. More observations of these stars in different regions of GCs, in particular determinations of masses and multiplicity fraction compared to the environment, will shed light on their origin. Better evolutionary GC models will help to interpret observations and to understand substantial differences observed among different GCs.
Acknowledgements. We thank an anonymous referee, whose thorough report with many relevant remarks and suggestions resulted in a substantial improvement of the presentation of our results. We also thank Slavek Rucinski for reading and commenting on the earlier version of the paper. This research was partly supported by the National Science Centre under the grant DEC2011/03/B/ST9/03299.

\section{References}

Baraffe, I., Chabrier, G., Alland, F., \& Hauschildt, P. H. 1998, A\&A, 337, 403 Barnes, S. A. 2009, in The Ages of Stars, eds. E. E. Mamajek, D. R. Soderblom, \& R. F. G. Wyse, IAU Symp., 258, 345

Barnes, S. A. 2011, in The Physics of Sun and Star Spots, eds. D. P. Choudhary, \& K. G. Strassmeier, IAU Symp., 273, 465

Beccari, G., Lützgendorf, N., Olczak, C., et al. 2012, ApJ, 754, 108

Bilir, S., Karataş, Y., Demircan, O., \& Eker, Z. 2005, MNRAS, 357, 497

Boffin, H. M. J., Carraro, G., \& Beccari, G. 2015, in Ecology of Blue Straggler Stars (Springer), Astrophys. Space Sci. Lib., 413

Bonnell, I. A. 1994, MNRAS, 269, 837

Boss, A. P. 1993, in The Realm of Interacting Binary Stars, eds. J. Sahade, G. E. McCluskey Jr., \& Y. Kondo (Dordrecht: Kluwer), 355

Chatterjee, S., Rasio, F. A., Sills, A., \& Glebbek, E. 2013, ApJ, 777, 106

Claret, A., \& Gimenez, A. 1992, A\&AS, 96, 255

Cranmer, S. R., \& Saar, S. H. 2011, ApJ, 741, 54

Dalessandro, E., Ferraro, F. R., Lanzoni, B., et al. 2013a, ApJ, 770, 45

Dalessandro, E., Ferraro, F. R., Massari, D., et al. 2013b, ApJ, 778, 135

De Marco, O., Shara, M. M., Zurek, D., et al. 2005, ApJ, 632, 894

Duchêne G., \& Kraus A. 2013, ARA\&A, 51, 269

Eggleton, P. P. 1983, ApJ, 268, 368

Eggleton, P. P., \& Kiseleva-Eggleton, L. 2002, ApJ, 575, 461

Eggleton, P. P., \& Kiseleva-Eggleton, L. 2006, Astrophys. Space Phys. Res., 304, 74

Fabrycky, D., \& Tremaine S. 2007, ApJ, 669, 1298

Ferraro, F. R., Sabbi, E., Gratton, R., et al. 2006, ApJ, 647, L53

Ferraro, F. R., Beccari, G., Dalessandro, E., et al. 2009, Nature, 462, 1028

Ferraro, F. R., Lanzoni, B., Dalessandro, E., et al. 2012, Nature, 492, 393

Ferraro, F. R., Lanzoni, B., Dalessandro, E., Mucciarelli, A., \& Lovisi, L. 2015, in Ecology of Blue Straggler Stars (Berlin, Heidelberg: Springer-Verlag), Astrophys. Space Sci. Lib., 413, 99

Fiorentino, G., Lanzoni, B., Dalessandro, E., et al. 2014, ApJ, 783, 34

Gazeas K., \& Stępień K. 2008, MNRAS, 390, 1577

Geller, A. M., \& Mathieu, R. D. 2011, Nature, 478, 356

Geller, A. M., \& Mathieu, R. D. 2012, AJ, 144, 54

Girardi, L., Bressan, A., Bertelli, G., \& Chiosi, C. 2000, A\&AS, 141, 371

Gosnell, N. M., Mathieu, R. D., Geller, A. M., et al. 2014, ApJ, 783, L8

Griffin, R. F. 1985, in Interacting Binaries, eds. P. P. Eggleton, \& J. E. Pringle (Dordrecht: Reidel), 1

Guinan, E. F., \& Bradstreet, D. H. 1988, in Formation and Evolution of Low Mass Stars, eds. A. K. Dupree, \& M. T. Logo (Dordrecht: Kluwer), 345

Han, Z., Tout, C. A., \& Eggleton P. P. 2000, MNRAS, 319, 215

Hebb, L., Stempels, H. C., Aigrain, S., et al. 2010, A\&A, 522, A37

Heggie, D. C. 1975, MNRAS, 173, 729

Helt, B. E., Jørgensen, H. E., King, S., \& Larsen, A. 1993, A\&A, 270, 297

Hills, J. G. 1975, AJ, 80, 809

Hut, R. 1983, ApJ, 272, L29

Hypki, A., \& Giersz, M. 2013, MNRAS, 429, 1221

Iben I. Jr. 1967, ApJ, 147, 624

Iben, I. Jr., \& Tutukov, A. V. 1984, ApJ, 284, 719

Kallrath, J., Milone, E. F., \& Stagg, C. R. 1992, ApJ, 389, 590

Kaluzny, J., \& Thompson, I. B. 2009, Acta Astron., 59, 273

Kaluzny, J., Olech, A., Thompson, I. B., et al. 2004, A\&A, 424, 1101

Kaluzny, J., Thompson, I. B., Rucinski S. M., et al. 2007, AJ, 134, 541

Kaluzny, J., Rozyczka, M., Thompson, I. B., \& Zloczewski, K. 2009, Acta Astron., 59, 371

Kaluzny, J., Thompson, I. B., Krzeminski, W., \& Zloczewski, K. 2010, Acta Astron., 60, 245

Kaluzny, J., Thompson, I. B., Rozyczka, M., \& Krzeminski, W. 2013a, Acta Astron., 63, 181

Kaluzny, J., Rozyczka, M., Pych, W., et al. 2013b, Acta Astron., 63, 309

Kratter, K. M., Matzner, C. D., Krumholz, M. R., \& Klein, R. I. 2010, ApJ, 708, 1585

Kroupa, P. 1995, MNRAS, 277, 1507

Kroupa, P, 2002, Science, 295, 82

Kuiper, G. P. 1941, ApJ, 93, 133

Leigh, N., \& Sills, A. 2011, MNRAS, 410, 2370

Leigh, N., Sills, A., \& Knigge, C. 2007, ApJ, 661, 210

Leigh, N., Sills, A., \& Knigge, C. 2011a, MNRAS, 415, 3771 
Leigh, N., Sills, A., \& Knigge, C. 2011b, MNRAS, 416, 1410 Leigh, N., Giersz, M., Webb, J. J., et al. 2013, MNRAS, 436, 3399 Li, K., \& Qian, S. 2012, AJ, 144, 161

Li, C., de Grus, R., Deng, L., \& Liu, X. 2013, ApJ, 770, L7

Liu, L., Qian, S.-B., \& Fernández-Lajús, E. 2011, MNRAS, 415, 1509

Lovisi, L. 2014, Mem. Soc. Astron. It., 85, 283

Lovisi, L., Mucciarelli, A., Ferraro, F. R., et al. 2010, ApJ, 719, L121

Lovisi, L., Mucciarelli, A., Lanzoni, B., et al. 2012, ApJ, 754, 91

Lovisi, L., Mucciarelli, A., Lanzoni, B., et al. 2013, ApJ, 772, 148

Machida, M. N., Tomisaka, K., Matsumoto, T., \& Inutsuka, S. 2008, ApJ, 677, 327

Machida, M. N., Inutsuka, S., \& Matsumoto, T. 2010, ApJ, 724, 1006

Mathieu, R. D. 1994, ARA\&A, 32, 465

Meibom, S., Barnes, S. A., Latham, D. W., et al. 2011, ApJ, 733, L9

Melo, C. H. F., Lovisio, E., Alcalá, J. M., \& Torres, G. 2001, A\&A, 378, 898

Miller, G. E., \& Scalo, J. M. 1979, ApJS, 41, 513

Milone, A. P., Piotto, G., Bedin, L. R., et al. 2012, A\&A, 540, A16

Mochnacki, S. W. 1981, ApJ, 245, 650

Moretti, A., De Angeli, F., \& Piotto, G. 2008, A\&A, 483, 183

Naoz, S., \& Fabrycky, D. C. 2014, ApJ, 793, 137

Nataf, D. M., Gould, A., \& Pinsonneault, M. H. 2012, Acta Astron., 62, 33

Nelson, C. A., \& Eggleton, P. P. 2001, ApJ, 552, 664

Ohtani, T., \& Tsuribe, T. 2013, PASJ, 65, 330

Paczyński, B. 1971, ARA\&A, 9, 183

Paczyński, B., Sienkiewicz, R., \& Szczygieł, D. M. 2007, MNRAS, 378, 961

Perets, H. B., \& Fabrycky, D. C. 2009, ApJ, 697, 1048

Pietrzyński, G., Thompson, I. B., Gieren, W., et al. 2012, Nature, 484, 75

Piotto, G., De Angeli, F., King, I. R., et al. 2004, ApJ, 604, L109

Prosser, C. F., Randich, S., Stauffer, J. R., Schmitt, J. H. M. M., \& Simon, T. 1996, AJ, 112, 1570

Raghavan, D., McAlister, H. A., Henry, T. J., et al. 2010, ApJS, 190, 1

Randich, S., Schmitt, J. H. M. M., Prosser C. F., \& Stauffer, J. R. 1996, A\&A, 305,785

Rasio, F. A. 1995, ApJ, 444, L41

Rasio, F. A., \& Shapiro, S. L. 1995, ApJ, 438, 887

Rozyczka, M., Kaluzny, J., Thompson, I. B., et al. 2013, Acta Astron., 63, 67

Rubenstein, E. P., \& Bailyn, C. D. 1996, AJ, 111, 260

Rucinski, S. M. 1998, AJ, 116, 2998

Rucinski, S. M. 2000, AJ, 120, 319

Rucinski, S. M. 2006, Ap\&SS, 304, 321
Rucinski, S. M. 2007, MNRAS, 382, 393

Rucinski, S. M. 2010, AIP Conf. Proc., 1314, 29

Rucinski, S. M., Pribulla, T., \& van Kerkwijk, M. H. 2007, AJ, 134, 2353

Rutten, R. G.M, \& Pylyser, E. 1988, A\&A, 191, 227

Salpeter, E. 1955, ApJ, 121, 161

Sandquist, E. L., Latham, D. W., Shetrone, M. D., \& Milone, A. A. E. 2003, AJ, 125,810

Sanna, N., Dalessandro, E., Ferraro, F. R., et al. 2014, ApJ, 780, 90

Sarna, M. 1993, MNRAS, 262, 534

Sarna, M. J. 2008, in Hot Subdwarf Stars and Related Objects, eds. U. Heber, S. Jeffery, \& R. Napiwotzki, ASP Conf. Ser., 392, 59

Sarna, M. J., \& De Greve J.-P. 1996, QJRAS, 37, 11

Scalo, J. M. 1986, FCPh, 11, 1

Schaller, G., Schaerer, D., Meynet, G., \& Maeder, A. 1992, A\&AS, 96, 269

Shara, M. M., Saffer, R. A., \& Livio, M. 1997, ApJ, 489, L59

Sills, A., Lombardi, J. C., Jr., Bailyn, C. D., et al. 1997, ApJ, 487, 290

Sills, A., Faber, J. A., Lombardi, J. C., Jr., et al. 2001, ApJ, 548, 323

Sills, A., Karakas, A., \& Lattanzio, J. 2009, ApJ, 692, 1411

Sills, A., Glebbek, E., Chatterjee, S., \& Rasio, F. A. 2013, ApJ, 777, 105

Stępień, K. 1995, MNRAS, 274, 1019

Stępień, K. 2006a, Acta Astron., 56, 199

Stępień, K. 2006b, Acta Astron., 56, 347

Stępień, K. 2009, MNRAS, 397, 857

Stępień, K. 2011a, Acta Astron., 61, 139

Stępień, K. 2011b, A\&A, 531, A18

Stẹpień, K., \& Gazeas, K. 2012, Acta Astron., 62, 153

Stępień, K., \& Kiraga, M. 2013a, Acta Astron., 63, 239

Stępień, K., \& Kiraga, M. 2013b, CEAB, 37, 381

Stȩpień, K., Schmitt, J. H. M. M., \& Voges, W. 2001, A\&A, 370, 157

Tian, B., Deng, L., Han, Z., \& Zhang, X. B. 2006, A\&A, 455, 247

Tognelli, E., Prada Moroni, P. G., \& Degl'Innocenti, S. 2011, A\&A, 533, A109

Tokovinin, A., Thomas, S., Sterzik, M., \& Udry, S. 2006, A\&A, 450, 681

Torres, G., Andersen, J., \& Gimenéz, A. 2010, A\&ARv, 18, 67

van den Berg, M., Orosz, J., Verbunt, F., \& Stassun, K. 2001, A\&A, 375, 375

van den Oord, G. H. J., \& Doyle, J. G. 1997, A\&A, 319, 578

Verbunt, F., \& Phinney, E. S. 1995, A\&A, 296, 709

Vorobyov, E. I. 2010, ApJ, 723, 1294

Webbink, R. F. 1976, ApJS, 32, 583

Wood, B. E., Müller, H. R., Zank, G. P., \& Linsky, J. L. 2002, ApJ, 574, 412

Pages 17 to 22 are available in the electronic edition of the journal at http://www . aanda.org 
K. Stępień and M. Kiraga: Blue stragglers and W UMa-type stars in globular clusters

Table 2. Model binaries with periods shorter than $1 \mathrm{~d}$ at the age of $11 \mathrm{Gyr}$.

\begin{tabular}{|c|c|c|c|c|c|c|c|}
\hline Model & $M_{1}\left(M_{\odot}\right)$ & $M_{2}\left(M_{\odot}\right)$ & $q$ & $P(\mathrm{~d})$ & $\log \left(L / L_{\odot}\right)$ & $\log T_{\mathrm{e}}$ & Remarks \\
\hline $0.75+0.4(1.6)$ & 0.458 & 0.618 & 0.741 & 0.168 & -0.489 & 3.723 & $\mathrm{CB}, \mathrm{MS}$ \\
\hline $0.75+0.5(1.5)$ & 0.526 & 0.643 & 0.818 & 0.177 & -0.380 & 3.731 & $\mathrm{CB}, \mathrm{MS}$ \\
\hline $0.75+0.7(1.5)$ & 0.580 & 0.764 & 0.759 & 0.194 & -0.149 & 3.757 & $\mathrm{CB}, \mathrm{MS}$ \\
\hline $0.8+0.4(1.8)$ & 0.441 & 0.678 & 0.650 & 0.173 & -0.363 & 3.737 & $\mathrm{CB}, \mathrm{MS}$ \\
\hline $0.8+0.5(1.7)$ & 0.544 & 0.667 & 0.816 & 0.226 & -0.295 & 3.737 & $\mathrm{CB}, \mathrm{MS}$ \\
\hline $0.8+0.6(1.6)$ & 0.547 & 0.752 & 0.727 & 0.220 & -0.154 & 3.754 & $\mathrm{CB}, \mathrm{MS}$ \\
\hline $0.8+0.7(1.6)$ & 0.535 & 0.850 & 0.629 & 0.213 & 0.017 & 3.777 & $\mathrm{CB}, \mathrm{MS}$ \\
\hline $0.81+0.5(1.7)$ & 0.487 & 0.732 & 0.665 & 0.201 & -0.223 & 3.750 & $\mathrm{CB}, \mathrm{MS}$ \\
\hline $0.81+0.6(1.6)$ & 0.489 & 0.818 & 0.598 & 0.198 & -0.057 & 3.770 & $\mathrm{CB}, \mathrm{MS}$ \\
\hline $0.82+0.4(1.9)$ & 0.447 & 0.690 & 0.648 & 0.183 & -0.327 & 3.740 & $\mathrm{CB}, \mathrm{MS}$ \\
\hline $0.82+0.5(1.7)$ & 0.460 & 0.767 & 0.600 & 0.166 & -0.166 & 3.759 & $\mathrm{CB}, \mathrm{MS}$ \\
\hline $0.82+0.7(1.7)$ & 0.659 & 0.742 & 0.888 & 0.283 & -0.085 & 3.751 & $\mathrm{CB}, \mathrm{MS}$ \\
\hline $0.83+0.4(1.9)$ & 0.432 & 0.713 & 0.606 & 0.145 & -0.284 & 3.745 & $\mathrm{CB}, \mathrm{MS}$ \\
\hline $0.83+0.5(1.8)$ & 0.525 & 0.710 & 0.739 & 0.222 & -0.217 & 3.746 & $\mathrm{CB}, \mathrm{MS}$ \\
\hline $0.83+0.6(1.7)$ & 0.552 & 0.771 & 0.716 & 0.240 & -0.101 & 3.758 & $\mathrm{CB}, \mathrm{MS}$ \\
\hline $0.83+0.7(1.7)$ & 0.584 & 0.827 & 0.706 & 0.256 & 0.008 & 3.769 & $\mathrm{CB}, \mathrm{MS}$ \\
\hline $0.84+0.4(2.0)$ & 0.442 & 0.711 & 0.622 & 0.193 & -0.282 & 3.745 & $\mathrm{CB}, \mathrm{MS}$ \\
\hline $0.84+0.5(1.8)$ & 0.451 & 0.792 & 0.569 & 0.205 & -0.122 & 3.765 & $\mathrm{CB}, \mathrm{MS}$ \\
\hline $0.84+0.6(1.7)$ & 0.481 & 0.852 & 0.565 & 0.219 & 0.011 & 3.778 & $\mathrm{CB}, \mathrm{MS}$ \\
\hline $0.84+0.7(1.7)$ & 0.521 & 0.898 & 0.580 & 0.229 & 0.117 & 3.788 & $\mathrm{CB}, \mathrm{MS}$ \\
\hline $0.85+0.5(1.9)$ & 0.542 & 0.710 & 0.763 & 0.237 & -0.192 & 3.746 & $\mathrm{CB}, \mathrm{MS}$ \\
\hline $0.85+0.6(1.8)$ & 0.611 & 0.731 & 0.836 & 0.271 & -0.108 & 3.750 & $\mathrm{CB}, \mathrm{MS}$ \\
\hline $0.86+0.4(2.1)$ & 0.415 & 0.755 & 0.550 & 0.190 & -0.217 & 3.755 & $\mathrm{CB}, \mathrm{MS}$ \\
\hline $0.86+0.5(1.9)$ & 0.452 & 0.808 & 0.559 & 0.214 & -0.086 & 3.769 & $\mathrm{CB}, \mathrm{MS}$ \\
\hline $0.86+0.6(1.8)$ & 0.507 & 0.843 & 0.601 & 0.244 & 0.010 & 3.775 & $\mathrm{CB}, \mathrm{MS}$ \\
\hline $0.86+0.7(1.8)$ & 0.589 & 0.847 & 0.695 & 0.275 & 0.055 & 3.772 & $\mathrm{CB}, \mathrm{MS}$ \\
\hline $0.865+0.4(2.2)$ & 0.474 & 0.700 & 0.677 & 0.226 & -0.261 & 3.743 & $\mathrm{CB}, \mathrm{MS}$ \\
\hline $0.865+0.5(1.9)$ & 0.444 & 0.821 & 0.541 & 0.176 & -0.055 & 3.772 & $\mathrm{CB}, \mathrm{MS}$ \\
\hline $0.865+0.5(2.0)$ & 0.556 & 0.709 & 0.784 & 0.261 & -0.172 & 3.746 & $\mathrm{CB}, \mathrm{MS}$ \\
\hline $0.865+0.6(1.8)$ & 0.478 & 0.875 & 0.546 & 0.214 & 0.063 & 3.784 & $\mathrm{CB}, \mathrm{MS}$ \\
\hline $0.865+0.7(1.8)$ & 0.527 & 0.913 & 0.577 & 0.260 & 0.148 & 3.790 & $\mathrm{CB}, \mathrm{MS}$ \\
\hline $0.865+0.8(1.9)$ & 0.662 & 0.863 & 0.767 & 0.305 & 0.117 & 3.773 & $\mathrm{CB}, \mathrm{MS}$ \\
\hline $0.8675+0.4(2.2)$ & 0.378 & 0.797 & 0.474 & 0.242 & -0.097 & 3.767 & $\mathrm{CB}, \mathrm{MS}$ \\
\hline $0.8675+0.4(2.3)$ & 0.432 & 0.744 & 0.581 & 0.292 & -0.123 & 3.752 & $\mathrm{CB}, \mathrm{MS}$ \\
\hline $0.8675+0.5(2.0)$ & 0.420 & 0.846 & 0.496 & 0.276 & 0.025 & 3.776 & $\mathrm{CB}, \mathrm{MS}$ \\
\hline $0.8675+0.5(2.1)$ & 0.508 & 0.758 & 0.670 & 0.345 & -0.023 & 3.754 & $\mathrm{CB}, \mathrm{MS}$ \\
\hline $0.8675+0.6(1.9)$ & 0.475 & 0.879 & 0.540 & 0.321 & 0.123 & 3.780 & $\mathrm{CB}, \mathrm{MS}$ \\
\hline $0.8675+0.6(2.0)$ & 0.595 & 0.759 & 0.784 & 0.412 & 0.079 & 3.754 & $\mathrm{CB}, \mathrm{MS}$ \\
\hline $0.8675+0.7(1.8)$ & 0.426 & 1.012 & 0.421 & 0.242 & 0.336 & 3.818 & $\mathrm{CB}, \mathrm{BS}$ \\
\hline $0.8675+0.7(1.9)$ & 0.537 & 0.903 & 0.595 & 0.372 & 0.204 & 3.781 & $\mathrm{CB}, \mathrm{MS}$ \\
\hline $0.8675+0.8(1.9)$ & 0.479 & 1.046 & 0.458 & 0.331 & 0.403 & 3.821 & $\mathrm{CB}, \mathrm{BS}$ \\
\hline $0.8675+0.8(2.0)$ & 0.593 & 0.934 & 0.635 & 0.489 & 0.318 & 3.780 & $\mathrm{CB}, \mathrm{MS}$ \\
\hline $0.88+0.5(2.0)$ & 0.191 & 1.072 & 0.178 & 0.845 & 0.543 & 3.823 & $\mathrm{NCB}, \mathrm{BS}$ \\
\hline $0.9+0.4(2.4)$ & 0.189 & 1.001 & 0.189 & 0.843 & 0.436 & 3.802 & $\mathrm{NCB}, \mathrm{MS}$ \\
\hline $0.9+0.6(1.9)$ & 0.152 & 1.221 & 0.124 & 0.709 & 0.836 & 3.887 & NCB, BS \\
\hline
\end{tabular}


Table 3. BS mergers formed between 10 and $11 \mathrm{Gyr}$, evolved to the age of $11 \mathrm{Gyr}$.

\begin{tabular}{|c|c|c|c|c|}
\hline Model & $t_{\text {merg }}$ (years) & $M\left(M_{\odot}\right)$ & $\log \left(L / L_{\odot}\right)$ & $\log T_{\mathrm{e}}$ \\
\hline $0.75+0.4(1.5)$ & $1.037 \mathrm{E}+10$ & 0.981 & 0.331 & 3.841 \\
\hline $0.8+0.4(1.7)$ & $1.040 \mathrm{E}+10$ & 1.024 & 0.467 & 3.857 \\
\hline $0.8+0.5(1.6)$ & $1.075 \mathrm{E}+10$ & 1.114 & 0.657 & 3.888 \\
\hline $0.8+0.6(1.5)$ & $1.042 \mathrm{E}+10$ & 1.204 & 0.971 & 3.912 \\
\hline $0.8+0.7(1.5)$ & $1.011 \mathrm{E}+10$ & 1.294 & 1.072 & 3.906 \\
\hline $0.81+0.4(1.7)$ & $1.005 \mathrm{E}+10$ & 1.033 & 0.529 & 3.862 \\
\hline $0.81+0.4(1.8)$ & $1.092 \mathrm{E}+10$ & 1.028 & 0.439 & 3.856 \\
\hline $0.81+0.5(1.6)$ & $1.044 \mathrm{E}+10$ & 1.122 & 0.728 & 3.891 \\
\hline $0.81+0.6(1.5)$ & $1.014 \mathrm{E}+10$ & 1.216 & 1.141 & 3.793 \\
\hline $0.82+0.4(1.8)$ & $1.057 \mathrm{E}+10$ & 1.039 & 0.505 & 3.862 \\
\hline $0.82+0.5(1.6)$ & $1.014 \mathrm{E}+10$ & 1.134 & 0.846 & 3.894 \\
\hline $0.82+0.6(1.6)$ & $1.086 \mathrm{E}+10$ & 1.217 & 0.856 & 3.910 \\
\hline $0.83+0.4(1.8)$ & $1.018 \mathrm{E}+10$ & 1.051 & 0.572 & 3.869 \\
\hline $0.83+0.5(1.7)$ & $1.072 \mathrm{E}+10$ & 1.138 & 0.734 & 3.895 \\
\hline $0.83+0.6(1.6)$ & $1.055 \mathrm{E}+10$ & 1.229 & 0.995 & 3.913 \\
\hline $0.84+0.4(1.9)$ & $1.059 \mathrm{E}+10$ & 1.056 & 0.547 & 3.869 \\
\hline $0.84+0.5(1.7)$ & $1.038 \mathrm{E}+10$ & 1.149 & 0.830 & 3.898 \\
\hline $0.84+0.6(1.6)$ & $1.024 \mathrm{E}+10$ & 1.241 & 1.251 & 3.748 \\
\hline $0.85+0.4(1.9)$ & $1.019 \mathrm{E}+10$ & 1.068 & 0.625 & 3.875 \\
\hline $0.85+0.4(2.0)$ & $1.099 \mathrm{E}+10$ & 1.061 & 0.528 & 3.868 \\
\hline $0.85+0.5(1.7)$ & $1.002 \mathrm{E}+10$ & 1.160 & 1.003 & 3.863 \\
\hline $0.85+0.5(1.8)$ & $1.087 \mathrm{E}+10$ & 1.153 & 0.749 & 3.899 \\
\hline $0.85+0.6(1.7)$ & $1.085 \mathrm{E}+10$ & 1.242 & 0.911 & 3.912 \\
\hline $0.85+0.7(1.7)$ & $1.085 \mathrm{E}+10$ & 1.330 & 1.048 & 3.921 \\
\hline $0.86+0.4(2.0)$ & $1.049 \mathrm{E}+10$ & 1.074 & 0.615 & 3.876 \\
\hline $0.86+0.5(1.8)$ & $1.046 \mathrm{E}+10$ & 1.165 & 0.867 & 3.905 \\
\hline $0.86+0.6(1.7)$ & $1.045 \mathrm{E}+10$ & 1.254 & 1.128 & 3.831 \\
\hline $0.86+0.7(1.7)$ & $1.053 \mathrm{E}+10$ & 1.340 & 1.341 & 3.733 \\
\hline $0.865+0.4(2.0)$ & $1.023 \mathrm{E}+10$ & 1.080 & 0.672 & 3.879 \\
\hline $0.865+0.4(2.1)$ & $1.099 \mathrm{E}+10$ & 1.074 & 0.566 & 3.874 \\
\hline $0.865+0.5(1.8)$ & $1.021 \mathrm{E}+10$ & 1.172 & 0.987 & 3.885 \\
\hline $0.865+0.6(1.7)$ & $1.022 \mathrm{E}+10$ & 1.262 & 1.133 & 3.829 \\
\hline $0.865+0.8(1.8)$ & $1.064 \mathrm{E}+10$ & 1.429 & 1.802 & 3.716 \\
\hline $0.8675+0.4(2.0)$ & $1.015 \mathrm{E}+10$ & 1.083 & 0.728 & 3.881 \\
\hline $0.8675+0.4(2.1)$ & $1.090 \mathrm{E}+10$ & 1.077 & 0.637 & 3.878 \\
\hline $0.8675+0.5(1.8)$ & $1.011 \mathrm{E}+10$ & 1.173 & 1.017 & 3.773 \\
\hline $0.8675+0.5(1.9)$ & $1.086 \mathrm{E}+10$ & 1.168 & 0.827 & 3.897 \\
\hline $0.8675+0.6(1.8)$ & $1.093 \mathrm{E}+10$ & 1.254 & 0.983 & 3.916 \\
\hline $0.87+0.4(2.0)$ & $1.005 \mathrm{E}+10$ & 1.086 & 0.772 & 3.880 \\
\hline $0.87+0.5(1.9)$ & $1.080 \mathrm{E}+10$ & 1.168 & 0.897 & 3.908 \\
\hline $0.88+0.4(2.1)$ & $1.031 \mathrm{E}+10$ & 1.092 & 0.822 & 3.878 \\
\hline $0.88+0.5(1.9)$ & $1.033 \mathrm{E}+10$ & 1.181 & 1.029 & 3.760 \\
\hline $0.88+0.6(1.8)$ & $1.041 \mathrm{E}+10$ & 1.271 & 1.275 & 3.739 \\
\hline $0.9+0.4(2.2)$ & $1.011 \mathrm{E}+10$ & 1.110 & 0.986 & 3.808 \\
\hline
\end{tabular}


K. Stępień and M. Kiraga: Blue stragglers and W UMa-type stars in globular clusters

Table 4. Model binaries with periods shorter than $1 \mathrm{~d}$ at the age of $12 \mathrm{Gyr}$.

\begin{tabular}{|c|c|c|c|c|c|c|c|}
\hline Model & $M_{1}\left(M_{\odot}\right)$ & $M_{2}\left(M_{\odot}\right)$ & $q$ & $P(\mathrm{~d})$ & $\log \left(L / L_{\odot}\right)$ & $\log T_{\mathrm{e}}$ & Remarks \\
\hline $0.7+0.4(1.5)$ & 0.445 & 0.583 & 0.763 & 0.142 & -0.528 & 3.720 & $\mathrm{CB}, \mathrm{MS}$ \\
\hline $0.75+0.5(1.6)$ & 0.541 & 0.619 & 0.874 & 0.199 & -0.398 & 3.728 & $\mathrm{CB}, \mathrm{MS}$ \\
\hline $0.75+0.7(1.6)$ & 0.603 & 0.731 & 0.825 & 0.223 & -0.194 & 3.749 & $\mathrm{CB}, \mathrm{MS}$ \\
\hline $0.8+0.4(1.9)$ & 0.426 & 0.686 & 0.621 & 0.167 & -0.348 & 3.739 & $\mathrm{CB}, \mathrm{MS}$ \\
\hline $0.8+0.5(1.8)$ & 0.545 & 0.657 & 0.830 & 0.229 & -0.287 & 3.736 & $\mathrm{CB}, \mathrm{MS}$ \\
\hline $0.8+0.6(1.7)$ & 0.552 & 0.738 & 0.748 & 0.229 & -0.161 & 3.751 & $\mathrm{CB}, \mathrm{MS}$ \\
\hline $0.8+0.7(1.7)$ & 0.533 & 0.842 & 0.633 & 0.231 & 0.002 & 3.775 & $\mathrm{CB}, \mathrm{MS}$ \\
\hline $0.81+0.5(1.8)$ & 0.468 & 0.743 & 0.630 & 0.206 & -0.203 & 3.752 & $\mathrm{CB}, \mathrm{MS}$ \\
\hline $0.81+0.6(1.7)$ & 0.471 & 0.827 & 0.570 & 0.215 & -0.044 & 3.773 & $\mathrm{CB}, \mathrm{MS}$ \\
\hline $0.81+0.7(1.7)$ & 0.511 & 0.872 & 0.586 & 0.187 & 0.070 & 3.782 & $\mathrm{CB}, \mathrm{MS}$ \\
\hline $0.82+0.4(2.0)$ & 0.410 & 0.718 & 0.571 & 0.183 & -0.290 & 3.746 & $\mathrm{CB}, \mathrm{MS}$ \\
\hline $0.82+0.5(1.8)$ & 0.439 & 0.780 & 0.563 & 0.162 & -0.141 & 3.762 & $\mathrm{CB}, \mathrm{MS}$ \\
\hline $0.82+0.6(1.8)$ & 0.602 & 0.705 & 0.854 & 0.280 & -0.155 & 3.746 & $\mathrm{CB}, \mathrm{MS}$ \\
\hline $0.82+0.7(1.8)$ & 0.634 & 0.759 & 0.835 & 0.295 & -0.066 & 3.754 & $\mathrm{CB}, \mathrm{MS}$ \\
\hline $0.83+0.4(2.1)$ & 0.458 & 0.679 & 0.675 & 0.208 & -0.312 & 3.738 & $\mathrm{CB}, \mathrm{MS}$ \\
\hline $0.83+0.5(1.9)$ & 0.486 & 0.742 & 0.655 & 0.223 & -0.183 & 3.752 & $\mathrm{CB}, \mathrm{MS}$ \\
\hline $0.83+0.6(1.8)$ & 0.526 & 0.788 & 0.668 & 0.248 & -0.071 & 3.762 & $\mathrm{CB}, \mathrm{MS}$ \\
\hline $0.83+0.7(1.8)$ & 0.565 & 0.836 & 0.676 & 0.264 & 0.026 & 3.771 & $\mathrm{CB}, \mathrm{MS}$ \\
\hline $0.84+0.4(2.1)$ & 0.398 & 0.747 & 0.533 & 0.183 & -0.236 & 3.753 & $\mathrm{CB}, \mathrm{MS}$ \\
\hline $0.84+0.5(1.9)$ & 0.424 & 0.811 & 0.523 & 0.198 & -0.086 & 3.770 & $\mathrm{CB}, \mathrm{MS}$ \\
\hline $0.84+0.6(1.8)$ & 0.457 & 0.866 & 0.528 & 0.220 & 0.041 & 3.782 & $\mathrm{CB}, \mathrm{MS}$ \\
\hline $0.84+0.7(1.8)$ & 0.498 & 0.911 & 0.547 & 0.236 & 0.138 & 3.791 & $\mathrm{CB}, \mathrm{MS}$ \\
\hline $0.85+0.4(2.2)$ & 0.435 & 0.717 & 0.607 & 0.209 & -0.252 & 3.746 & $\mathrm{CB}, \mathrm{MS}$ \\
\hline $0.85+0.5(2.0)$ & 0.487 & 0.756 & 0.644 & 0.239 & -0.141 & 3.755 & $\mathrm{CB}, \mathrm{MS}$ \\
\hline $0.85+0.6(1.9)$ & 0.565 & 0.766 & 0.738 & 0.273 & -0.067 & 3.756 & $\mathrm{CB}, \mathrm{MS}$ \\
\hline $0.85+0.7(1.9)$ & 0.650 & 0.769 & 0.845 & 0.312 & -0.007 & 3.756 & $\mathrm{CB}, \mathrm{MS}$ \\
\hline $0.86+0.4(2.2)$ & 0.391 & 0.772 & 0.506 & 0.166 & -0.178 & 3.760 & $\mathrm{CB}, \mathrm{MS}$ \\
\hline $0.86+0.4(2.3)$ & 0.459 & 0.704 & 0.652 & 0.228 & -0.248 & 3.744 & $\mathrm{CB}, \mathrm{MS}$ \\
\hline $0.86+0.5(2.0)$ & 0.420 & 0.834 & 0.504 & 0.200 & -0.035 & 3.776 & $\mathrm{CB}, \mathrm{MS}$ \\
\hline $0.86+0.5(2.1)$ & 0.537 & 0.714 & 0.752 & 0.277 & -0.159 & 3.747 & $\mathrm{CB}, \mathrm{MS}$ \\
\hline $0.86+0.6(1.9)$ & 0.466 & 0.873 & 0.534 & 0.248 & 0.064 & 3.783 & $\mathrm{CB}, \mathrm{MS}$ \\
\hline $0.86+0.7(1.9)$ & 0.543 & 0.883 & 0.615 & 0.279 & 0.109 & 3.781 & $\mathrm{CB}, \mathrm{MS}$ \\
\hline $0.865+0.4(2.3)$ & 0.413 & 0.753 & 0.548 & 0.214 & -0.197 & 3.754 & $\mathrm{CB}, \mathrm{MS}$ \\
\hline $0.865+0.5(2.1)$ & 0.485 & 0.771 & 0.629 & 0.256 & -0.103 & 3.758 & $\mathrm{CB}, \mathrm{MS}$ \\
\hline $0.865+0.6(1.9)$ & 0.452 & 0.891 & 0.507 & 0.196 & 0.100 & 3.789 & $\mathrm{CB}, \mathrm{MS}$ \\
\hline $0.865+0.6(2.0)$ & 0.587 & 0.755 & 0.777 & 0.314 & -0.030 & 3.754 & $\mathrm{CB}, \mathrm{MS}$ \\
\hline $0.865+0.7(1.9)$ & 0.485 & 0.944 & 0.514 & 0.267 & 0.204 & 3.799 & $\mathrm{CB}, \mathrm{MS}$ \\
\hline $0.865+0.8(2.0)$ & 0.574 & 0.939 & 0.611 & 0.311 & 0.221 & 3.791 & $\mathrm{CB}, \mathrm{MS}$ \\
\hline $0.8675+0.4(2.3)$ & 0.215 & 0.952 & 0.226 & 0.388 & 0.229 & 3.804 & $\mathrm{CB}, \mathrm{MS}$ \\
\hline $0.8675+0.4(2.4)$ & 0.233 & 0.931 & 0.250 & 0.553 & 0.244 & 3.790 & $\mathrm{NCB}, \mathrm{MS}$ \\
\hline $0.8675+0.4(2.5)$ & 0.275 & 0.887 & 0.310 & 0.705 & 0.262 & 3.775 & $\mathrm{NCB}, \mathrm{MS}$ \\
\hline $0.8675+0.4(2.6)$ & 0.283 & 0.877 & 0.323 & 0.955 & 0.352 & 3.768 & NCB, MS \\
\hline $0.8675+0.5(2.1)$ & 0.221 & 1.032 & 0.214 & 0.583 & 0.418 & 3.819 & $\mathrm{NCB}, \mathrm{BS}$ \\
\hline $0.8675+0.5(2.2)$ & 0.279 & 0.972 & 0.287 & 0.711 & 0.377 & 3.792 & $\mathrm{NCB}, \mathrm{MS}$ \\
\hline $0.8675+0.6(1.9)$ & 0.179 & 1.161 & 0.154 & 0.579 & 0.668 & 3.872 & $\mathrm{NCB}, \mathrm{BS}$ \\
\hline $0.8675+0.6(2.0)$ & 0.256 & 1.080 & 0.237 & 0.790 & 0.552 & 3.819 & $\mathrm{NCB}, \mathrm{BS}$ \\
\hline $0.8675+0.7(1.9)$ & 0.224 & 1.199 & 0.187 & 0.655 & 0.750 & 3.873 & $\mathrm{NCB}, \mathrm{BS}$ \\
\hline $0.8675+0.7(2.0)$ & 0.296 & 1.126 & 0.263 & 0.924 & 0.645 & 3.821 & $\mathrm{NCB}, \mathrm{BS}$ \\
\hline $0.8675+0.8(1.9)$ & 0.148 & 1.363 & 0.109 & 0.630 & 1.078 & 3.920 & $\mathrm{NCB}, \mathrm{BS}$ \\
\hline $0.8675+0.8(2.0)$ & 0.275 & 1.229 & 0.224 & 0.940 & 0.816 & 3.858 & $\mathrm{NCB}, \mathrm{BS}$ \\
\hline
\end{tabular}


A\&A 577, A117 (2015)

Table 5. BS mergers formed between 10 and 12 Gyr, evolved to the age of $12 \mathrm{Gyr}$

\begin{tabular}{|c|c|c|c|c|}
\hline Model & $t_{\text {merg }}$ (years) & $M\left(M_{\odot}\right)$ & $\log \left(L / L_{\odot}\right)$ & $\log T_{\mathrm{e}}$ \\
\hline $0.75+0.4(1.5)$ & $1.037 \mathrm{E}+10$ & 0.981 & 0.418 & 3.846 \\
\hline $0.8+0.4(1.7)$ & $1.040 \mathrm{E}+10$ & 1.024 & 0.584 & 3.862 \\
\hline $0.8+0.5(1.6)$ & $1.075 E+10$ & 1.114 & 0.864 & 3.881 \\
\hline $0.81+0.4(1.7)$ & $1.005 E+10$ & 1.033 & 0.678 & 3.863 \\
\hline $0.81+0.4(1.8)$ & $1.092 \mathrm{E}+10$ & 1.028 & 0.546 & 3.862 \\
\hline $0.82+0.4(1.8)$ & $1.057 \mathrm{E}+10$ & 1.039 & 0.635 & 3.866 \\
\hline $0.83+0.4(1.8)$ & $1.018 \mathrm{E}+10$ & 1.051 & 0.743 & 3.867 \\
\hline $0.84+0.4(1.9)$ & $1.059 \mathrm{E}+10$ & 1.056 & 0.694 & 3.871 \\
\hline $0.85+0.5(1.8)$ & $1.087 \mathrm{E}+10$ & 1.153 & 1.054 & 3.781 \\
\hline $0.86+0.4(2.0)$ & $1.049 \mathrm{E}+10$ & 1.074 & 0.809 & 3.869 \\
\hline $0.865+0.4(2.0)$ & $1.023 \mathrm{E}+10$ & 1.080 & 0.940 & 3.817 \\
\hline $0.865+0.4(2.1)$ & $1.099 \mathrm{E}+10$ & 1.074 & 0.715 & 3.878 \\
\hline $0.8675+0.4(2.0)$ & $1.015 \mathrm{E}+10$ & 1.083 & 1.468 & 3.721 \\
\hline $0.8675+0.4(2.1)$ & $1.090 \mathrm{E}+10$ & 1.077 & 0.858 & 3.860 \\
\hline $0.87+0.4(2.0)$ & $1.005 E+10$ & 1.086 & 0.965 & 3.845 \\
\hline $0.87+0.5(1.9)$ & $1.080 \mathrm{E}+10$ & 1.168 & 1.054 & 3.819 \\
\hline $0.75+0.4(1.6)$ & $1.146 \mathrm{E}+10$ & 0.974 & 0.327 & 3.840 \\
\hline $0.75+0.5(1.5)$ & $1.143 E+10$ & 1.065 & 0.538 & 3.870 \\
\hline $0.75+0.6(1.5)$ & $1.194 \mathrm{E}+10$ & 1.148 & 0.683 & 3.898 \\
\hline $0.75+0.7(1.5)$ & $1.126 \mathrm{E}+10$ & 1.242 & 1.085 & 3.893 \\
\hline $0.8+0.4(1.8)$ & $1.133 \mathrm{E}+10$ & 1.016 & 0.476 & 3.856 \\
\hline $0.8+0.5(1.7)$ & $1.178 \mathrm{E}+10$ & 1.105 & 0.646 & 3.885 \\
\hline $0.8+0.6(1.6)$ & $1.149 \mathrm{E}+10$ & 1.195 & 0.945 & 3.913 \\
\hline $0.81+0.4(1.9)$ & $1.183 E+10$ & 1.021 & 0.448 & 3.855 \\
\hline $0.81+0.5(1.7)$ & $1.143 \mathrm{E}+10$ & 1.116 & 0.733 & 3.889 \\
\hline $0.81+0.6(1.6)$ & $1.117 \mathrm{E}+10$ & 1.206 & 1.117 & 3.791 \\
\hline $0.82+0.4(1.9)$ & $1.141 \mathrm{E}+10$ & 1.034 & 0.521 & 3.862 \\
\hline $0.82+0.5(1.7)$ & $1.108 \mathrm{E}+10$ & 1.127 & 0.859 & 3.888 \\
\hline $0.82+0.6(1.7)$ & $1.188 \mathrm{E}+10$ & 1.208 & 0.845 & 3.907 \\
\hline $0.82+0.7(1.7)$ & $1.173 E+10$ & 1.294 & 1.053 & 3.923 \\
\hline $0.83+0.4(1.9)$ & $1.103 E+10$ & 1.045 & 0.595 & 3.868 \\
\hline $0.83+0.4(2.0)$ & $1.187 \mathrm{E}+10$ & 1.038 & 0.498 & 3.861 \\
\hline $0.83+0.5(1.8)$ & $1.167 \mathrm{E}+10$ & 1.130 & 0.743 & 3.893 \\
\hline $0.83+0.6(1.7)$ & $1.154 \mathrm{E}+10$ & 1.219 & 1.017 & 3.911 \\
\hline $0.83+0.7(1.7)$ & $1.144 \mathrm{E}+10$ & 1.307 & 1.461 & 3.726 \\
\hline $0.84+0.4(2.0)$ & $1.144 \mathrm{E}+10$ & 1.050 & 0.573 & 3.868 \\
\hline $0.84+0.5(1.8)$ & $1.129 \mathrm{E}+10$ & 1.141 & 0.873 & 3.894 \\
\hline $0.84+0.6(1.7)$ & $1.120 \mathrm{E}+10$ & 1.230 & 1.436 & 3.724 \\
\hline $0.85+0.4(2.1)$ & $1.180 \mathrm{E}+10$ & 1.056 & 0.559 & 3.869 \\
\hline $0.85+0.5(1.9)$ & $1.177 \mathrm{E}+10$ & 1.146 & 0.772 & 3.896 \\
\hline $0.85+0.6(1.8)$ & $1.178 \mathrm{E}+10$ & 1.234 & 0.965 & 3.918 \\
\hline $0.85+0.7(1.8)$ & $1.183 E+10$ & 1.319 & 1.049 & 3.919 \\
\hline $0.86+0.4(2.1)$ & $1.127 \mathrm{E}+10$ & 1.068 & 0.651 & 3.875 \\
\hline $0.86+0.5(1.9)$ & $1.130 \mathrm{E}+10$ & 1.158 & 0.936 & 3.891 \\
\hline $0.86+0.6(1.8)$ & $1.137 \mathrm{E}+10$ & 1.246 & 1.206 & 3.754 \\
\hline $0.86+0.7(1.8)$ & $1.149 \mathrm{E}+10$ & 1.331 & 1.430 & 3.727 \\
\hline $0.865+0.4(2.2)$ & $1.178 \mathrm{E}+10$ & 1.069 & 0.600 & 3.875 \\
\hline $0.865+0.5(1.9)$ & $1.105 E+10$ & 1.164 & 1.006 & 3.783 \\
\hline $0.865+0.5(2.0)$ & $1.189 \mathrm{E}+10$ & 1.157 & 0.784 & 3.898 \\
\hline $0.8675+0.4(2.2)$ & $1.158 \mathrm{E}+10$ & 1.070 & 0.723 & 3.875 \\
\hline $0.8675+0.5(2.0)$ & $1.158 \mathrm{E}+10$ & 1.161 & 0.966 & 3.886 \\
\hline $0.87+0.4(2.1)$ & $1.148 \mathrm{E}+10$ & 1.068 & 1.369 & 3.723 \\
\hline $0.87+0.4(2.2)$ & $1.179 \mathrm{E}+10$ & 1.055 & 0.910 & 3.754 \\
\hline $0.87+0.6(1.8)$ & $1.162 \mathrm{E}+10$ & 1.238 & 0.890 & 3.755 \\
\hline $0.88+0.4(2.2)$ & $1.151 \mathrm{E}+10$ & 1.069 & 1.152 & 3.739 \\
\hline $0.89+0.4(2.2)$ & $1.123 E+10$ & 1.088 & 1.036 & 3.746 \\
\hline $0.89+0.4(2.3)$ & $1.144 \mathrm{E}+10$ & 1.076 & 1.110 & 3.741 \\
\hline
\end{tabular}


K. Stępień and M. Kiraga: Blue stragglers and W UMa-type stars in globular clusters

Table 6. Model binaries with periods shorter than $1 \mathrm{~d}$ at the age of $13 \mathrm{Gyr}$.

\begin{tabular}{|c|c|c|c|c|c|c|c|}
\hline Model & $M_{1}\left(M_{\odot}\right)$ & $M_{2}\left(M_{\odot}\right)$ & $q$ & $P(\mathrm{~d})$ & $\log \left(L / L_{\odot}\right)$ & $\log T_{\mathrm{e}}$ & Remarks \\
\hline $0.7+0.5(1.5)$ & 0.480 & 0.633 & 0.758 & 0.142 & -0.418 & 3.729 & $\mathrm{CB}, \mathrm{MS}$ \\
\hline $0.7+0.6(1.5)$ & 0.518 & 0.680 & 0.762 & 0.178 & -0.326 & 3.738 & $\mathrm{CB}, \mathrm{MS}$ \\
\hline $0.75+0.6(1.6)$ & 0.509 & 0.732 & 0.695 & 0.185 & -0.230 & 3.750 & $\mathrm{CB}, \mathrm{MS}$ \\
\hline $0.8+0.4(2.0)$ & 0.408 & 0.699 & 0.584 & 0.167 & -0.328 & 3.742 & $\mathrm{CB}, \mathrm{MS}$ \\
\hline $0.8+0.5(1.9)$ & 0.528 & 0.667 & 0.792 & 0.236 & -0.276 & 3.738 & $\mathrm{CB}, \mathrm{MS}$ \\
\hline $0.8+0.6(1.8)$ & 0.565 & 0.716 & 0.789 & 0.241 & -0.171 & 3.747 & $\mathrm{CB}, \mathrm{MS}$ \\
\hline $0.8+0.7(1.8)$ & 0.551 & 0.816 & 0.675 & 0.245 & -0.026 & 3.768 & $\mathrm{CB}, \mathrm{MS}$ \\
\hline $0.81+0.4(2.1)$ & 0.446 & 0.668 & 0.668 & 0.199 & -0.338 & 3.736 & $\mathrm{CB}, \mathrm{MS}$ \\
\hline $0.81+0.5(1.9)$ & 0.450 & 0.754 & 0.597 & 0.210 & -0.186 & 3.755 & $\mathrm{CB}, \mathrm{MS}$ \\
\hline $0.81+0.6(1.8)$ & 0.468 & 0.822 & 0.569 & 0.225 & -0.047 & 3.771 & $\mathrm{CB}, \mathrm{MS}$ \\
\hline $0.81+0.7(1.8)$ & 0.493 & 0.881 & 0.560 & 0.210 & 0.080 & 3.785 & $\mathrm{CB}, \mathrm{MS}$ \\
\hline $0.82+0.4(2.1)$ & 0.386 & 0.737 & 0.524 & 0.179 & -0.256 & 3.751 & $\mathrm{CB}, \mathrm{MS}$ \\
\hline $0.82+0.5(1.9)$ & 0.410 & 0.802 & 0.511 & 0.170 & -0.101 & 3.769 & $\mathrm{CB}, \mathrm{MS}$ \\
\hline $0.82+0.5(2.0)$ & 0.537 & 0.675 & 0.796 & 0.253 & -0.220 & 3.740 & $\mathrm{CB}, \mathrm{MS}$ \\
\hline $0.82+0.6(1.9)$ & 0.589 & 0.709 & 0.831 & 0.287 & -0.132 & 3.746 & $\mathrm{CB}, \mathrm{MS}$ \\
\hline $0.82+0.7(1.9)$ & 0.636 & 0.747 & 0.851 & 0.307 & -0.048 & 3.752 & $\mathrm{CB}, \mathrm{MS}$ \\
\hline $0.83+0.4(2.2)$ & 0.413 & 0.717 & 0.576 & 0.204 & -0.266 & 3.746 & $\mathrm{CB}, \mathrm{MS}$ \\
\hline $0.83+0.5(2.0)$ & 0.448 & 0.771 & 0.581 & 0.227 & -0.141 & 3.759 & $\mathrm{CB}, \mathrm{MS}$ \\
\hline $0.83+0.6(1.9)$ & 0.500 & 0.806 & 0.620 & 0.255 & -0.043 & 3.766 & $\mathrm{CB}, \mathrm{MS}$ \\
\hline $0.83+0.7(1.9)$ & 0.541 & 0.849 & 0.637 & 0.277 & 0.051 & 3.774 & $\mathrm{CB}, \mathrm{MS}$ \\
\hline $0.84+0.4(2.2)$ & 0.375 & 0.763 & 0.491 & 0.163 & -0.199 & 3.758 & $\mathrm{CB}, \mathrm{MS}$ \\
\hline $0.84+0.4(2.3)$ & 0.449 & 0.690 & 0.651 & 0.228 & -0.264 & 3.741 & $\mathrm{CB}, \mathrm{MS}$ \\
\hline $0.84+0.5(2.0)$ & 0.398 & 0.830 & 0.480 & 0.192 & -0.045 & 3.776 & $\mathrm{CB}, \mathrm{MS}$ \\
\hline $0.84+0.5(2.1)$ & 0.521 & 0.707 & 0.737 & 0.263 & -0.168 & 3.746 & $\mathrm{CB}, \mathrm{MS}$ \\
\hline $0.84+0.6(1.9)$ & 0.429 & 0.884 & 0.485 & 0.222 & 0.077 & 3.787 & $\mathrm{CB}, \mathrm{MS}$ \\
\hline $0.84+0.6(2.0)$ & 0.611 & 0.704 & 0.868 & 0.324 & -0.084 & 3.746 & $\mathrm{CB}, \mathrm{MS}$ \\
\hline $0.84+0.7(1.9)$ & 0.465 & 0.934 & 0.498 & 0.249 & 0.182 & 3.798 & $\mathrm{CB}, \mathrm{MS}$ \\
\hline $0.85+0.4(2.3)$ & 0.383 & 0.764 & 0.501 & 0.204 & -0.191 & 3.758 & $\mathrm{CB}, \mathrm{MS}$ \\
\hline $0.85+0.4(2.4)$ & 0.461 & 0.685 & 0.673 & 0.265 & -0.246 & 3.741 & $\mathrm{CB}, \mathrm{MS}$ \\
\hline $0.85+0.5(2.1)$ & 0.438 & 0.795 & 0.551 & 0.240 & -0.085 & 3.765 & $\mathrm{CB}, \mathrm{MS}$ \\
\hline $0.85+0.6(2.0)$ & 0.514 & 0.809 & 0.635 & 0.281 & -0.011 & 3.766 & $\mathrm{CB}, \mathrm{MS}$ \\
\hline $0.85+0.7(2.0)$ & 0.600 & 0.808 & 0.743 & 0.322 & 0.047 & 3.763 & $\mathrm{CB}, \mathrm{MS}$ \\
\hline $0.86+0.4(2.4)$ & 0.402 & 0.753 & 0.534 & 0.221 & -0.190 & 3.755 & $\mathrm{CB}, \mathrm{MS}$ \\
\hline $0.86+0.5(2.2)$ & 0.486 & 0.756 & 0.643 & 0.270 & -0.109 & 3.755 & $\mathrm{CB}, \mathrm{MS}$ \\
\hline $0.86+0.6(2.0)$ & 0.422 & 0.908 & 0.465 & 0.250 & 0.127 & 3.793 & $\mathrm{CB}, \mathrm{MS}$ \\
\hline $0.86+0.6(2.1)$ & 0.612 & 0.717 & 0.854 & 0.333 & -0.038 & 3.748 & $\mathrm{CB}, \mathrm{MS}$ \\
\hline $0.86+0.7(2.0)$ & 0.498 & 0.916 & 0.544 & 0.289 & 0.168 & 3.790 & $\mathrm{CB}, \mathrm{MS}$ \\
\hline $0.865+0.4(2.4)$ & 0.346 & 0.811 & 0.427 & 0.220 & -0.092 & 3.771 & $\mathrm{CB}, \mathrm{MS}$ \\
\hline $0.865+0.4(2.5)$ & 0.405 & 0.753 & 0.538 & 0.274 & -0.137 & 3.754 & $\mathrm{CB}, \mathrm{MS}$ \\
\hline $0.865+0.4(2.6)$ & 0.398 & 0.760 & 0.524 & 0.414 & -0.041 & 3.754 & $\mathrm{CB}, \mathrm{MS}$ \\
\hline $0.865+0.5(2.2)$ & 0.403 & 0.844 & 0.477 & 0.267 & 0.012 & 3.776 & $\mathrm{CB}, \mathrm{MS}$ \\
\hline $0.865+0.5(2.3)$ & 0.496 & 0.750 & 0.661 & 0.342 & -0.039 & 3.753 & $\mathrm{CB}, \mathrm{MS}$ \\
\hline $0.865+0.5(2.4)$ & 0.523 & 0.725 & 0.721 & 0.524 & 0.084 & 3.750 & $\mathrm{NCB}, \mathrm{MS}$ \\
\hline $0.865+0.6(2.1)$ & 0.478 & 0.857 & 0.558 & 0.328 & 0.091 & 3.775 & $\mathrm{CB}, \mathrm{MS}$ \\
\hline $0.865+0.6(2.2)$ & 0.508 & 0.827 & 0.614 & 0.516 & 0.158 & 3.763 & $\mathrm{NCB}, \mathrm{MS}$ \\
\hline $0.865+0.7(2.0)$ & 0.411 & 1.007 & 0.408 & 0.281 & 0.322 & 3.817 & $\mathrm{CB}, \mathrm{BS}$ \\
\hline $0.865+0.7(2.1)$ & 0.501 & 0.916 & 0.547 & 0.451 & 0.254 & 3.781 & $\mathrm{CB}, \mathrm{MS}$ \\
\hline $0.865+0.8(2.1)$ & 0.470 & 1.031 & 0.456 & 0.330 & 0.378 & 3.818 & $\mathrm{CB}, \mathrm{BS}$ \\
\hline $0.865+0.7(2.2)$ & 0.627 & 0.792 & 0.792 & 0.617 & 0.253 & 3.757 & NCB, MS \\
\hline $0.865+0.8(2.2)$ & 0.527 & 0.975 & 0.541 & 0.584 & 0.389 & 3.786 & $\mathrm{NCB}, \mathrm{MS}$ \\
\hline $0.8675+0.4(2.4)$ & 0.125 & 1.023 & 0.122 & 0.806 & 0.443 & 3.818 & $\mathrm{NCB}, \mathrm{BS}$ \\
\hline $0.8675+0.5(2.1)$ & 0.104 & 1.133 & 0.092 & 0.875 & 0.691 & 3.860 & $\mathrm{NCB}, \mathrm{BS}$ \\
\hline
\end{tabular}


Table 7. BS mergers formed between 10 and $13 \mathrm{Gyr}$, evolved to the age of $13 \mathrm{Gyr}$.

\begin{tabular}{|c|c|c|c|c|c|c|c|c|c|}
\hline Model & $t_{\text {merg }}$ (years) & $M\left(M_{\odot}\right)$ & $\log \left(L / L_{\odot}\right)$ & $\log T_{\mathrm{e}}$ & model & $t_{\text {merg }}$ (years) & $M\left(M_{\odot}\right)$ & $\log \left(L / L_{\odot}\right)$ & $\log T_{\mathrm{e}}$ \\
\hline $0.75+0.4(1.5)$ & $1.037 \mathrm{E}+10$ & 0.981 & 0.533 & 3.849 & $0.8+0.4(1.9)$ & $1.225 \mathrm{E}+10$ & 1.010 & 0.479 & 3.855 \\
\hline $0.8+0.4(1.7)$ & $1.040 \mathrm{E}+10$ & 1.024 & 0.793 & 3.842 & $0.8+0.5(1.8)$ & $1.286 \mathrm{E}+10$ & 1.096 & 0.635 & 3.883 \\
\hline $0.85+0.5(1.8)$ & $1.087 \mathrm{E}+10$ & 1.153 & 0.899 & 3.785 & $0.8+0.6(1.7)$ & $1.260 \mathrm{E}+10$ & 1.185 & 0.872 & 3.905 \\
\hline $0.75+0.4(1.6)$ & $1.146 \mathrm{E}+10$ & 0.974 & 0.415 & 3.844 & $0.8+0.7(1.7)$ & $1.229 \mathrm{E}+10$ & 1.272 & 1.506 & 3.726 \\
\hline $0.75+0.5(1.5)$ & $1.143 E+10$ & 1.065 & 0.676 & 3.875 & $0.81+0.4(2.0)$ & $1.274 \mathrm{E}+10$ & 1.016 & 0.460 & 3.855 \\
\hline $0.75+0.6(1.5)$ & $1.194 \mathrm{E}+10$ & 1.148 & 0.896 & 3.893 & $0.81+0.5(1.8)$ & $1.244 \mathrm{E}+10$ & 1.107 & 0.731 & 3.887 \\
\hline $0.8+0.4(1.8)$ & $1.133 \mathrm{E}+10$ & 1.016 & 0.600 & 3.860 & $0.81+0.6(1.7)$ & $1.224 \mathrm{E}+10$ & 1.196 & 1.082 & 3.844 \\
\hline $0.8+0.5(1.7)$ & $1.178 \mathrm{E}+10$ & 1.105 & 0.847 & 3.879 & $0.82+0.4(2.0)$ & $1.231 \mathrm{E}+10$ & 1.026 & 0.532 & 3.861 \\
\hline $0.81+0.4(1.9)$ & $1.183 E+10$ & 1.021 & 0.560 & 3.860 & $0.82+0.5(1.8)$ & $1.203 \mathrm{E}+10$ & 1.119 & 0.867 & 3.883 \\
\hline $0.81+0.5(1.7)$ & $1.143 \mathrm{E}+10$ & 1.116 & 0.906 & 3.778 & $0.82+0.6(1.8)$ & $1.292 \mathrm{E}+10$ & 1.200 & 0.834 & 3.905 \\
\hline $0.82+0.4(1.9)$ & $1.141 \mathrm{E}+10$ & 1.034 & 0.662 & 3.864 & $0.82+0.7(1.8)$ & $1.278 \mathrm{E}+10$ & 1.285 & 1.016 & 3.910 \\
\hline $0.83+0.4(1.9)$ & $1.103 \mathrm{E}+10$ & 1.045 & 0.795 & 3.856 & $0.83+0.4(2.1)$ & $1.271 \mathrm{E}+10$ & 1.032 & 0.517 & 3.862 \\
\hline $0.83+0.4(2.0)$ & $1.187 \mathrm{E}+10$ & 1.038 & 0.624 & 3.866 & $0.83+0.5(1.9)$ & $1.260 \mathrm{E}+10$ & 1.123 & 0.754 & 3.890 \\
\hline $0.83+0.5(1.8)$ & $1.167 \mathrm{E}+10$ & 1.130 & 1.304 & 3.749 & $0.83+0.6(1.8)$ & $1.255 \mathrm{E}+10$ & 1.209 & 1.013 & 3.904 \\
\hline $0.84+0.4(2.0)$ & $1.144 \mathrm{E}+10$ & 1.050 & 0.746 & 3.866 & $0.83+0.7(1.8)$ & $1.247 \mathrm{E}+10$ & 1.297 & 1.309 & 3.731 \\
\hline $0.85+0.4(2.1)$ & $1.180 \mathrm{E}+10$ & 1.056 & 0.714 & 3.871 & $0.84+0.4(2.1)$ & $1.223 \mathrm{E}+10$ & 1.044 & 0.599 & 3.868 \\
\hline $0.85+0.5(1.9)$ & $1.177 \mathrm{E}+10$ & 1.146 & 0.923 & 3.804 & $0.84+0.5(1.9)$ & $1.219 \mathrm{E}+10$ & 1.134 & 0.903 & 3.884 \\
\hline $0.86+0.4(2.1)$ & $1.127 \mathrm{E}+10$ & 1.068 & 0.902 & 3.831 & $0.85+0.4(2.2)$ & $1.260 \mathrm{E}+10$ & 1.049 & 0.585 & 3.869 \\
\hline $0.86+0.5(1.9)$ & $1.130 \mathrm{E}+10$ & 1.158 & 0.885 & 3.831 & $0.85+0.5(2.0)$ & $1.265 \mathrm{E}+10$ & 1.137 & 0.798 & 3.891 \\
\hline $0.865+0.4(2.2)$ & $1.178 \mathrm{E}+10$ & 1.069 & 0.783 & 3.871 & $0.85+0.6(1.9)$ & $1.274 \mathrm{E}+10$ & 1.225 & 0.981 & 3.911 \\
\hline $0.865+0.5(1.9)$ & $1.105 \mathrm{E}+10$ & 1.164 & 0.775 & 3.870 & $0.85+0.7(1.9)$ & $1.281 \mathrm{E}+10$ & 1.309 & 1.062 & 3.925 \\
\hline $0.865+0.5(2.0)$ & $1.189 \mathrm{E}+10$ & 1.157 & 0.949 & 3.817 & $0.86+0.4(2.2)$ & $1.208 \mathrm{E}+10$ & 1.062 & 0.698 & 3.874 \\
\hline $0.8675+0.4(2.2)$ & $1.158 \mathrm{E}+10$ & 1.070 & 1.835 & 3.704 & $0.86+0.4(2.3)$ & $1.282 \mathrm{E}+10$ & 1.056 & 0.581 & 3.870 \\
\hline $0.8675+0.5(2.0)$ & $1.158 \mathrm{E}+10$ & 1.161 & 1.728 & 3.708 & $0.86+0.5(2.0)$ & $1.215 \mathrm{E}+10$ & 1.152 & 1.016 & 3.828 \\
\hline $0.87+0.4(2.1)$ & $1.148 \mathrm{E}+10$ & 1.068 & 1.811 & 3.705 & $0.86+0.5(2.1)$ & $1.300 \mathrm{E}+10$ & 1.143 & 0.758 & 3.896 \\
\hline $0.87+0.4(2.2)$ & $1.179 \mathrm{E}+10$ & 1.055 & 1.656 & 3.710 & $0.86+0.6(1.9)$ & $1.230 \mathrm{E}+10$ & 1.236 & 1.540 & 3.720 \\
\hline $0.88+0.4(2.2)$ & $1.151 \mathrm{E}+10$ & 1.069 & 1.823 & 3.704 & $0.86+0.7(1.9)$ & $1.244 \mathrm{E}+10$ & 1.321 & 2.126 & 3.700 \\
\hline $0.89+0.4(2.2)$ & $1.123 \mathrm{E}+10$ & 1.088 & 2.048 & 3.696 & $0.865+0.4(2.3)$ & $1.254 \mathrm{E}+10$ & 1.062 & 0.638 & 3.874 \\
\hline $0.7+0.4(1.5)$ & $1.226 \mathrm{E}+10$ & 0.927 & 0.246 & 3.829 & $0.865+0.5(2.1)$ & $1.271 \mathrm{E}+10$ & 1.151 & 0.834 & 3.895 \\
\hline $0.75+0.4(1.7)$ & $1.261 \mathrm{E}+10$ & 0.968 & 0.316 & 3.839 & $0.865+0.6(2.0)$ & $1.297 \mathrm{E}+10$ & 1.233 & 0.965 & 3.918 \\
\hline $0.75+0.5(1.6)$ & $1.269 \mathrm{E}+10$ & 1.056 & 0.504 & 3.866 & $0.8675+0.4(2.3)$ & $1.276 \mathrm{E}+10$ & 1.057 & 0.916 & 3.807 \\
\hline $0.75+0.7(1.6)$ & $1.252 \mathrm{E}+10$ & 1.230 & 0.919 & 3.912 & $0.8675+0.6(1.9)$ & $1.254 \mathrm{E}+10$ & 1.233 & 0.881 & 3.806 \\
\hline
\end{tabular}

